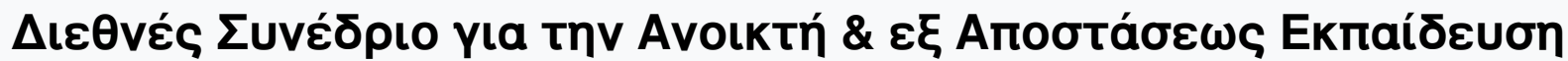

Tón. 6, Ap. 1A (2011)

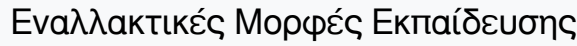

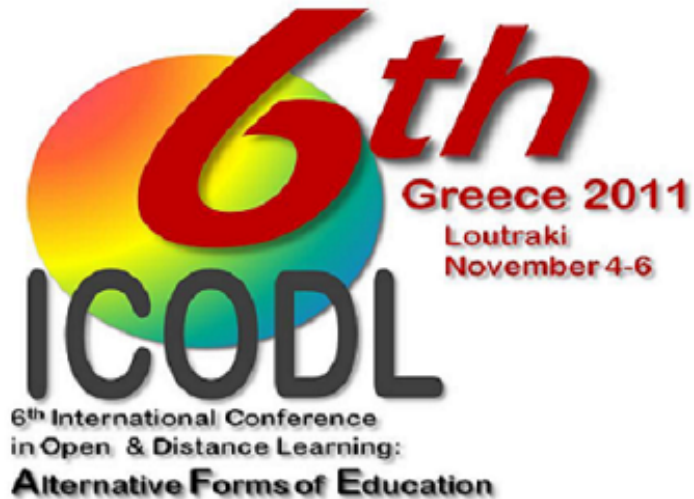

TOMOEA PART/MEPOE A

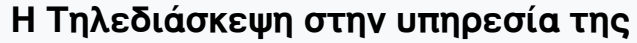

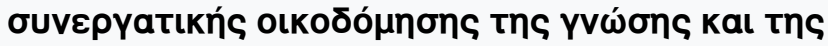

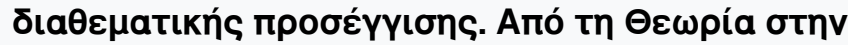

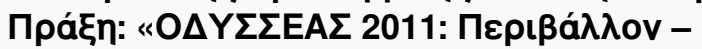

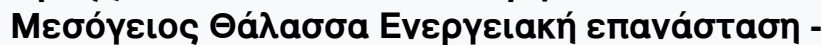

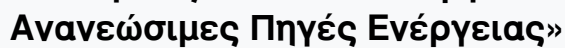

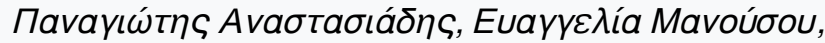

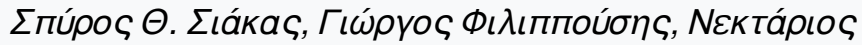

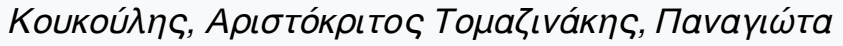

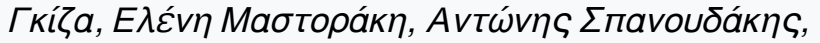

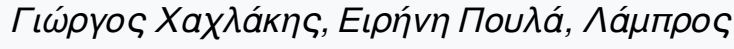

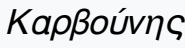

doi: $\underline{10.12681 / \text { icodl. } 685}$ 


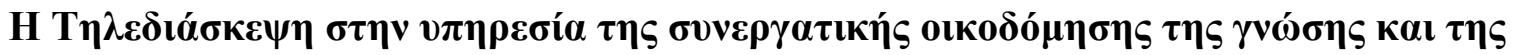

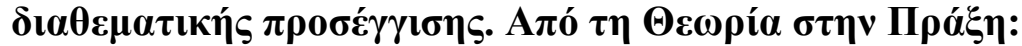

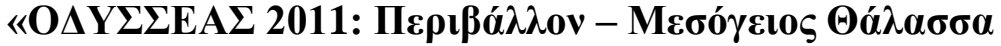

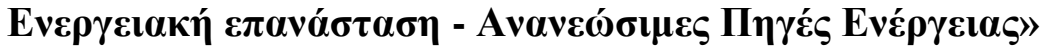

\section{Videoconferce and collaborative knowledge construction. From Theory to Practice: "ODYSSEUS 2011: Environment - Mediterranean Sea "KAPETAN SOS" Energy revolution - Renewable Energy"}

\begin{tabular}{|c|c|c|}
\hline & 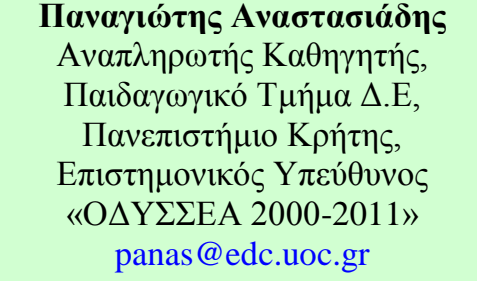 & \\
\hline 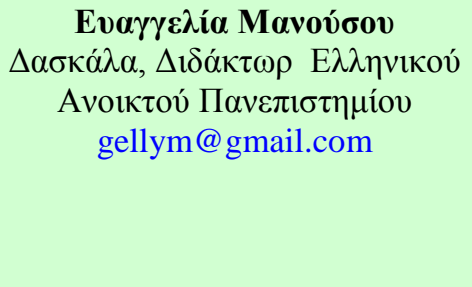 & 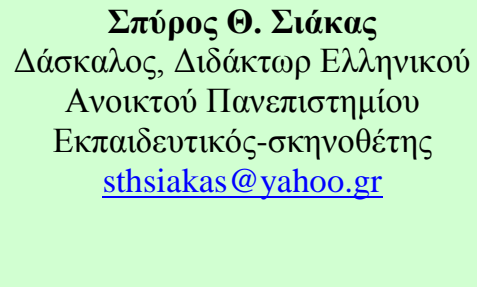 & 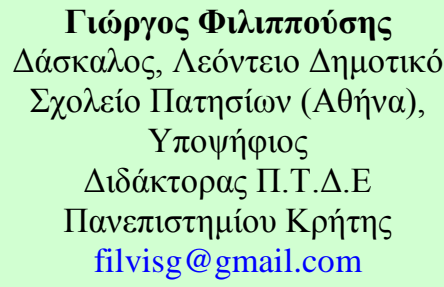 \\
\hline 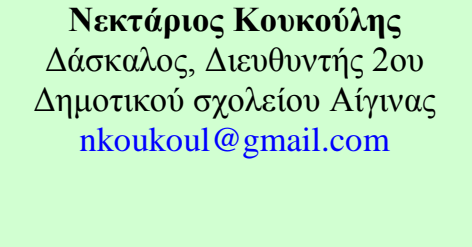 & 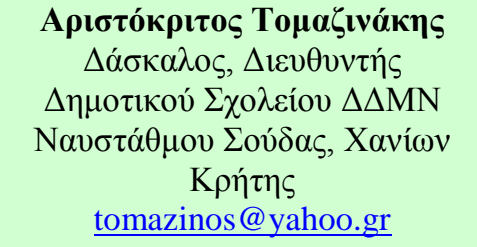 & 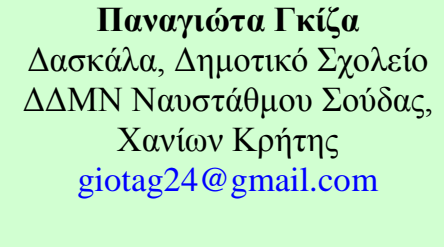 \\
\hline 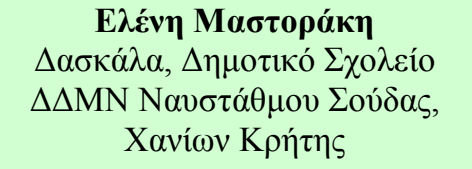 & 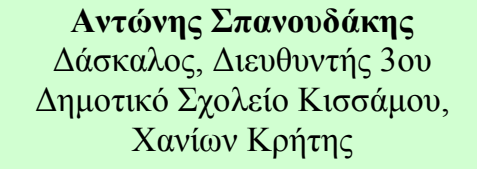 & 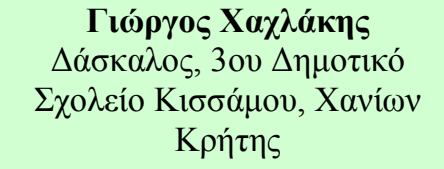 \\
\hline 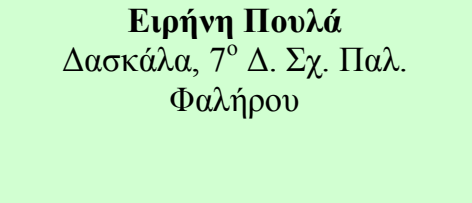 & & 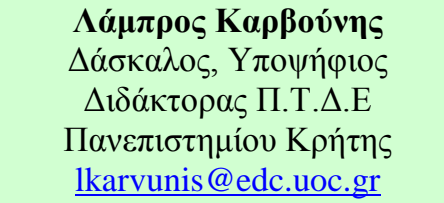 \\
\hline
\end{tabular}

\footnotetext{
Abstract

The aim of this paper is to present the design, implementation and evaluation of the methodology which focuses on the pedagogical utilization of Interactive Videoconferencing (IVC) in the contemporary elementary school.
} 
As part of the project "ODYSSEAS", during the school year 2010 - 2011, 152 students and 13 teachers from four elementary schools in Athens, Aigina and Chania collaborated at distance via IVC and, with the aid of the animation technique and distance learning material, designed and implemented constructive activities on the topic: "Environment Mediterranean Sea -Renewable Energy Sources. Major contributors for the development of the program 'ODYSSEUS' are the Department of Education in the University of Crete (http://www.edc.uoc.gr/ odysseas/ ).

\section{Пврі́ $\eta \psi \eta$}

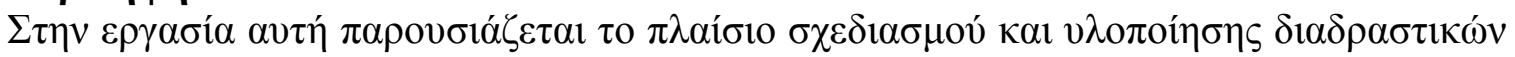

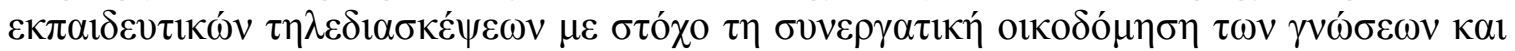

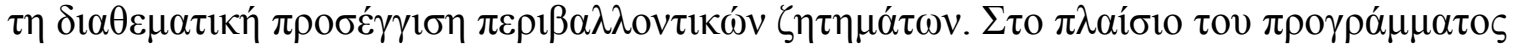

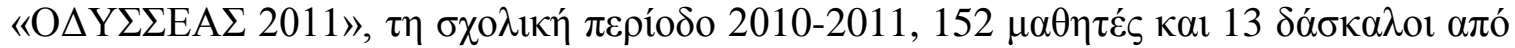

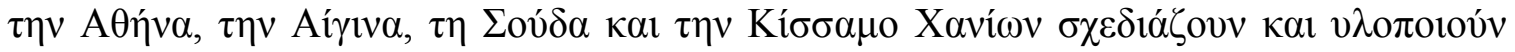

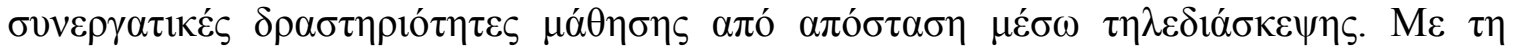

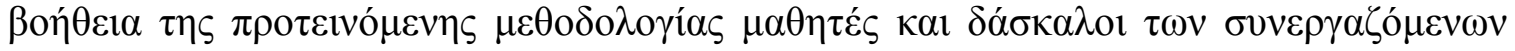

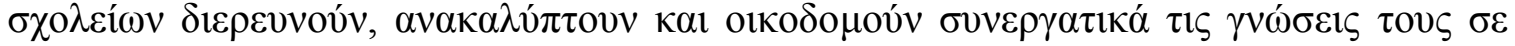

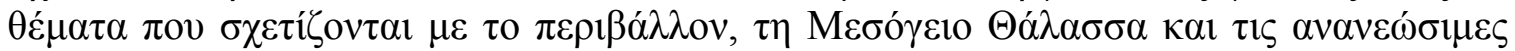

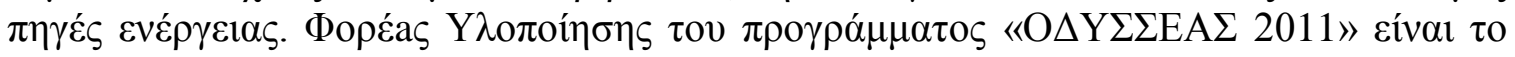

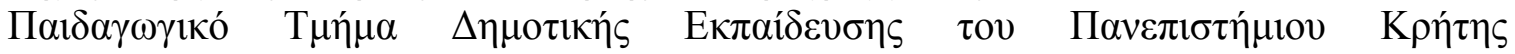
(http://www.edc.uoc.gr/ odysseas/ ).

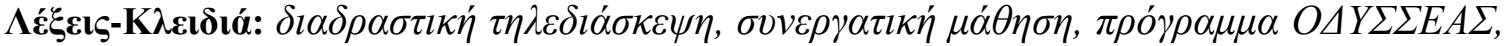

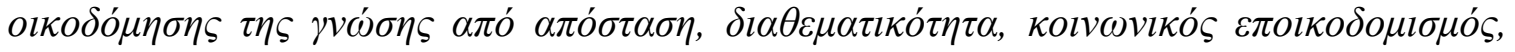

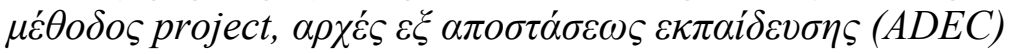

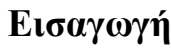

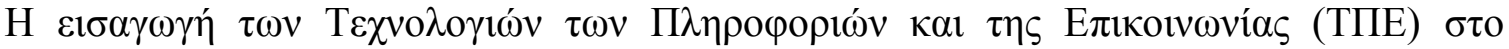

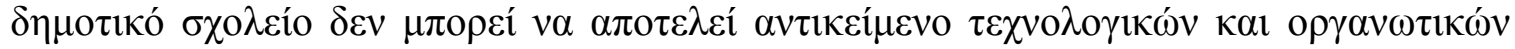

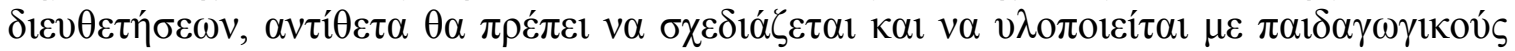

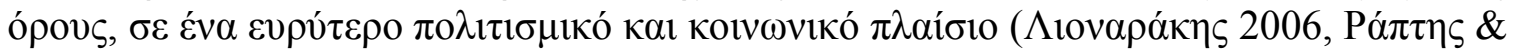

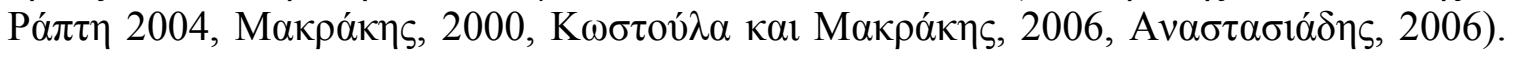

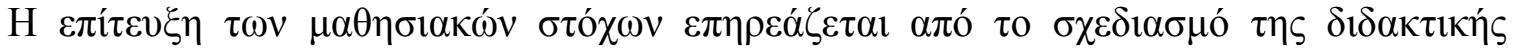

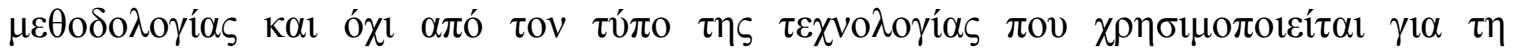

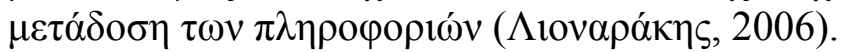

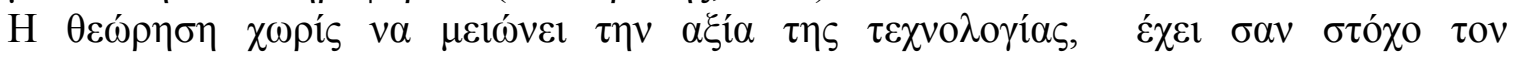

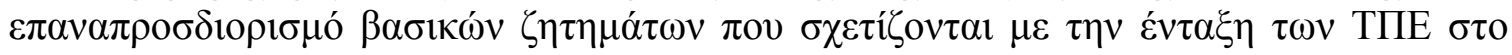

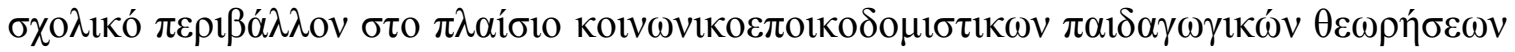

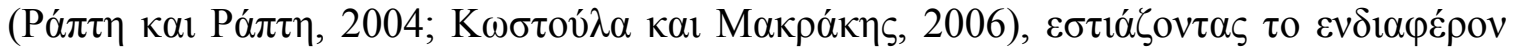

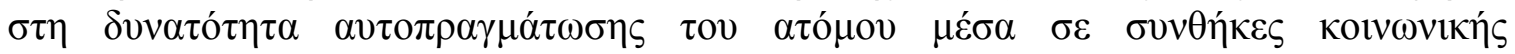

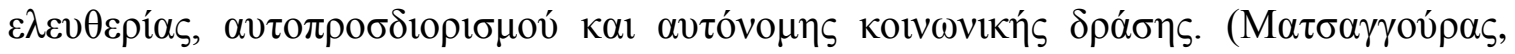
1999:103).

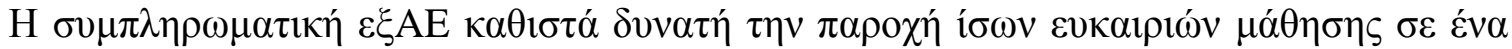

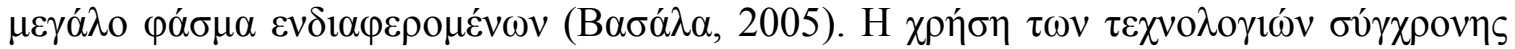

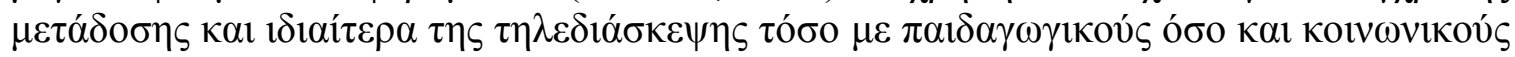

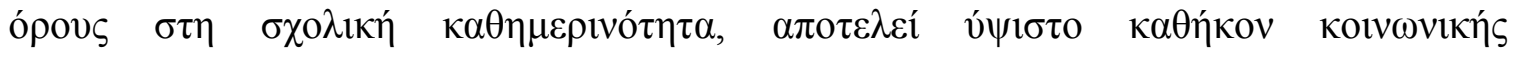


$6^{\text {th }}$ International Conference in Open \& Distance Learning - November 2011, Loutraki, Greece - PROCEEDINGS

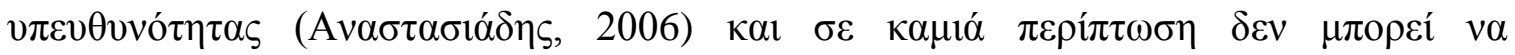

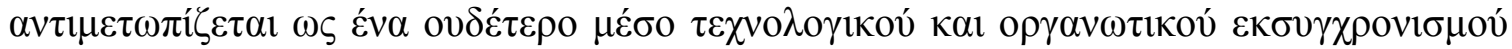

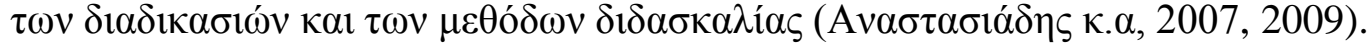

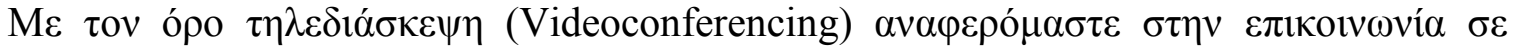

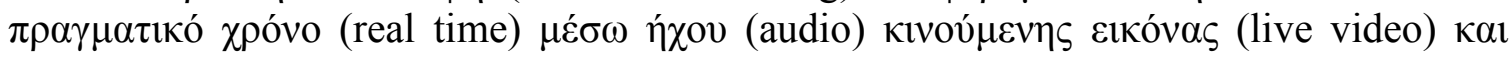

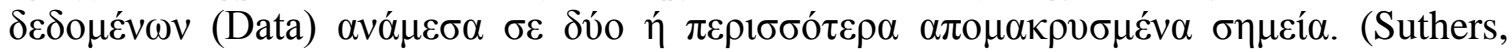
2001; Gibson אal Cohen, 2003; Reed \& Woodruff, 1995, Alexander et al., 1999;

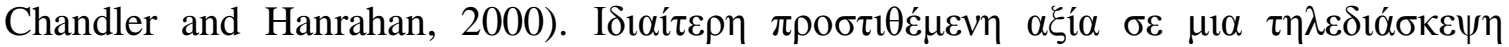

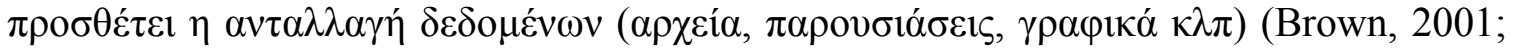

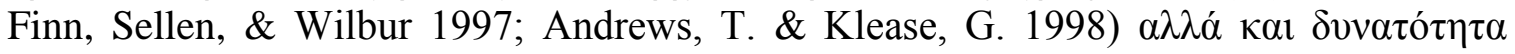

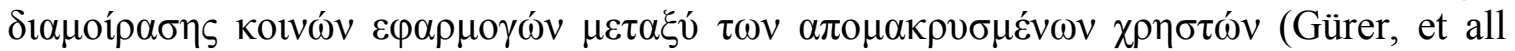
1999).

$\mathrm{H} \Delta \iota \alpha \delta \rho \alpha \sigma \tau \imath \kappa \eta ́ ~ T \eta \lambda \varepsilon \delta i \alpha ́ \sigma \kappa \varepsilon \psi \eta(\Delta \mathrm{T})$ - Interactive Videoconferencing (IVC) $\varepsilon \pi \imath \tau \rho \varepsilon ́ \pi \varepsilon \imath \sigma \varepsilon$

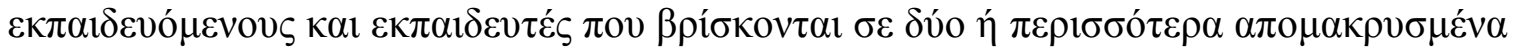

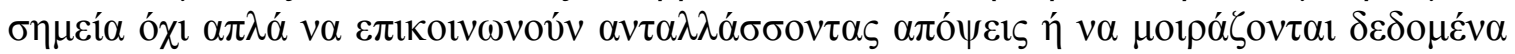

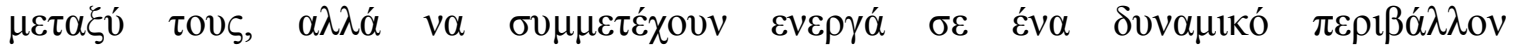

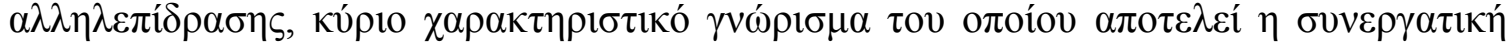

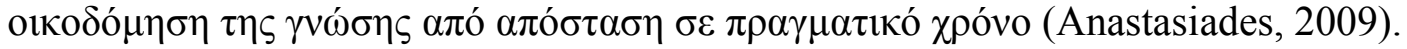

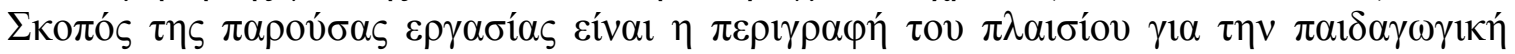

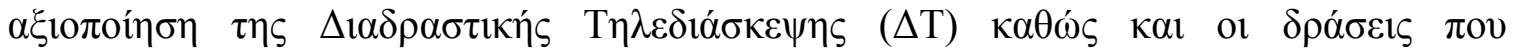

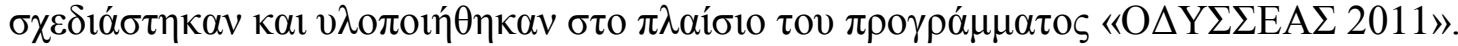

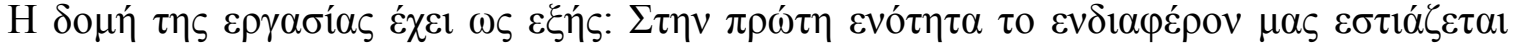

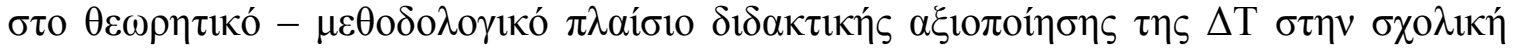

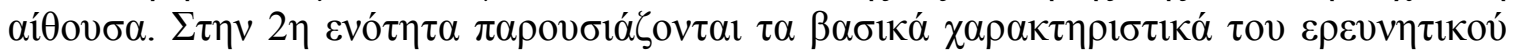

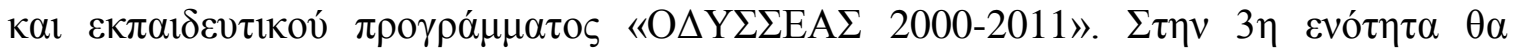

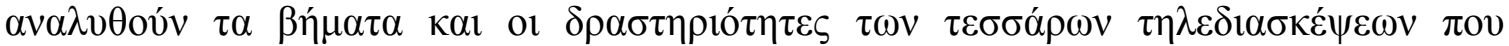

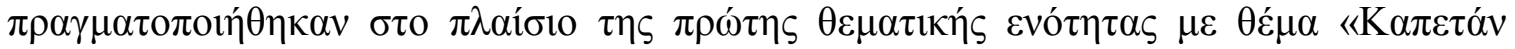
$\Sigma \mathrm{O} \Sigma-\mathrm{M \varepsilon \sigma ó} \gamma \varepsilon \operatorname{los} \Theta \alpha \dot{\lambda} \alpha \sigma \sigma \alpha »$.

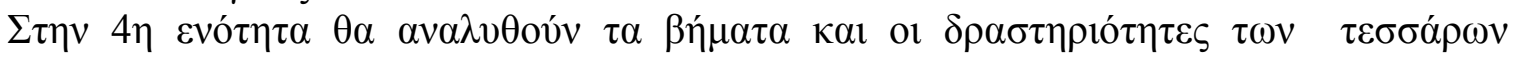

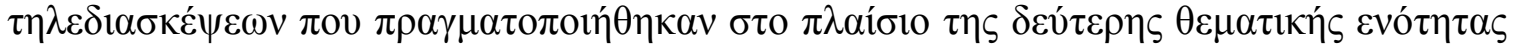

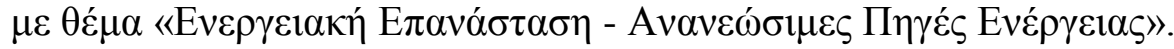

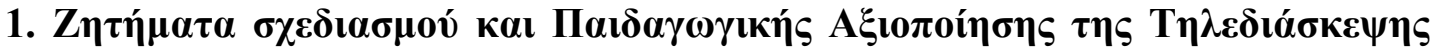

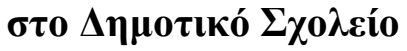

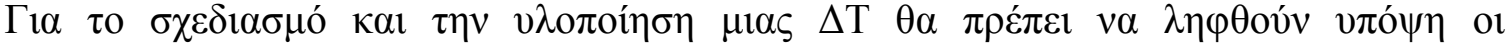

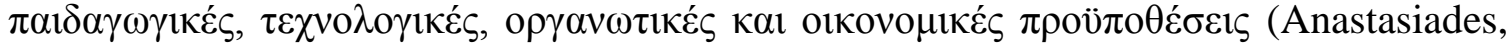
2009).

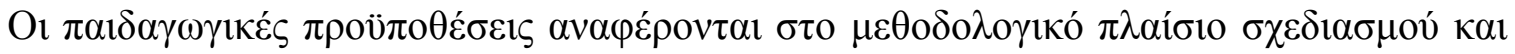

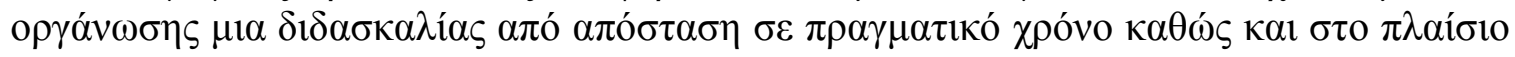

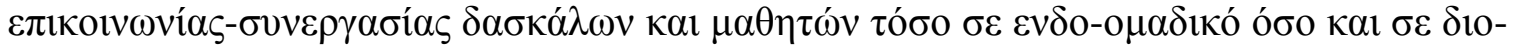

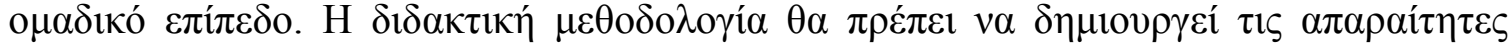

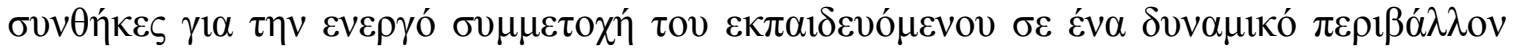

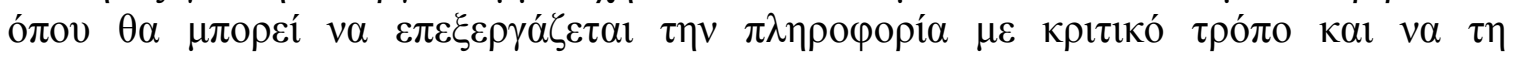
$\mu \varepsilon \tau o v \sigma i \omega ́ v \varepsilon ı ~ \sigma \varepsilon \gamma v \omega ́ \sigma \eta ~(A l l y, ~ 2004)$.

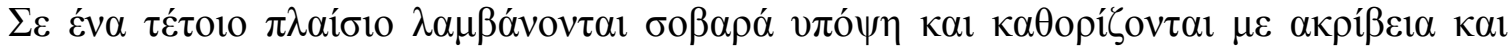

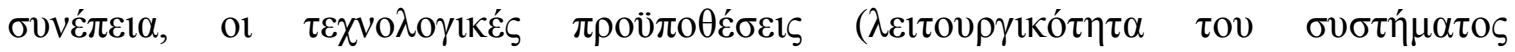




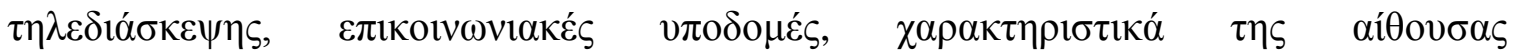

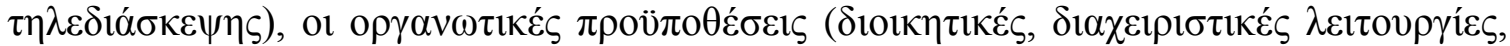

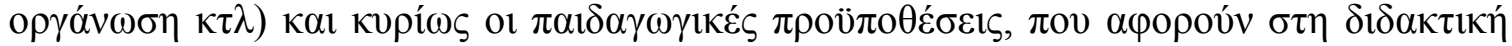

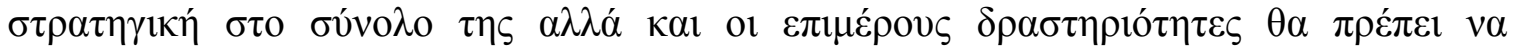

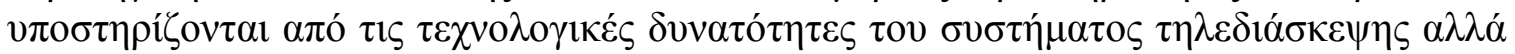

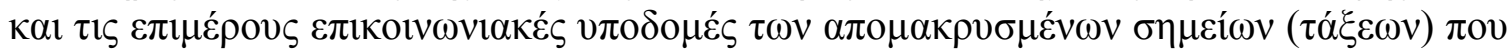

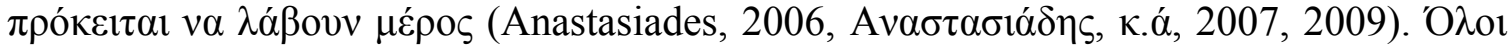

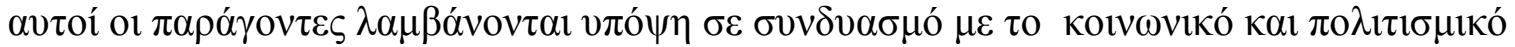

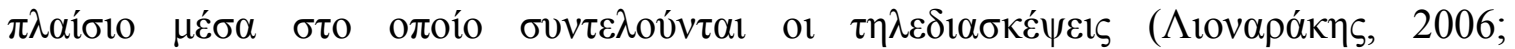

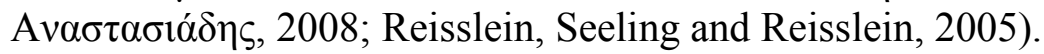

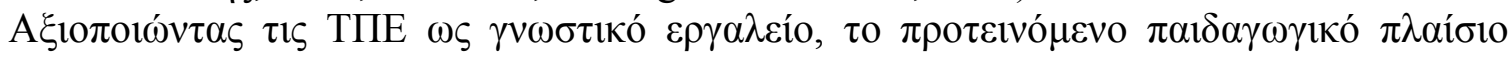

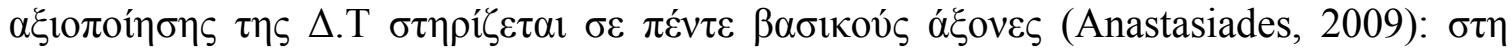

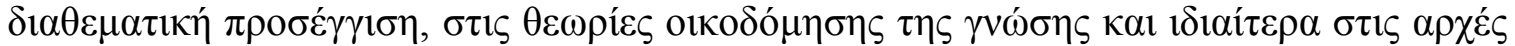

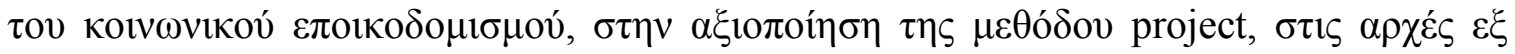

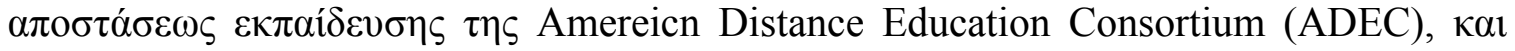

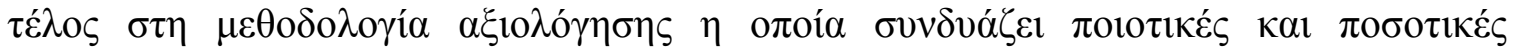

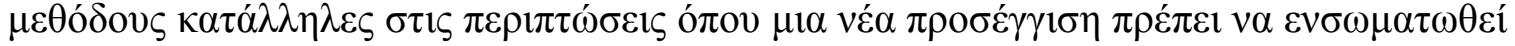

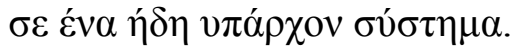

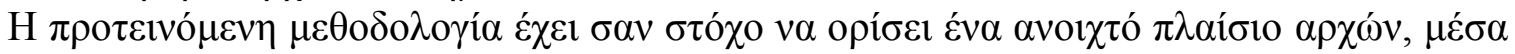

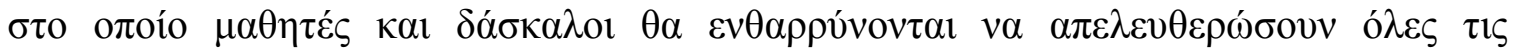

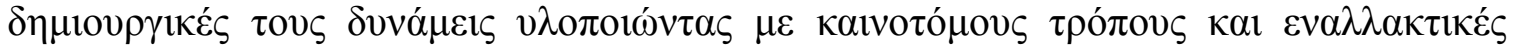

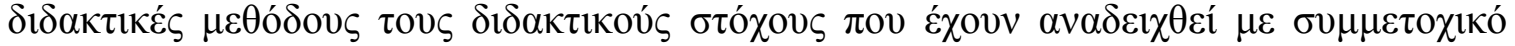

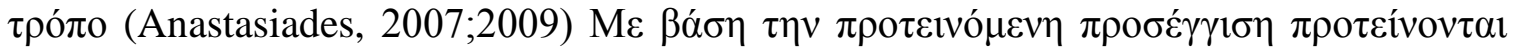

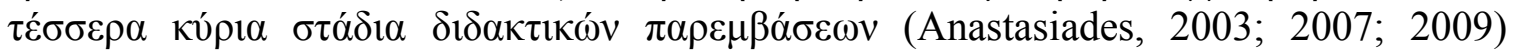
( $\Sigma \chi \underline{\mu} \mu \alpha 1)$.

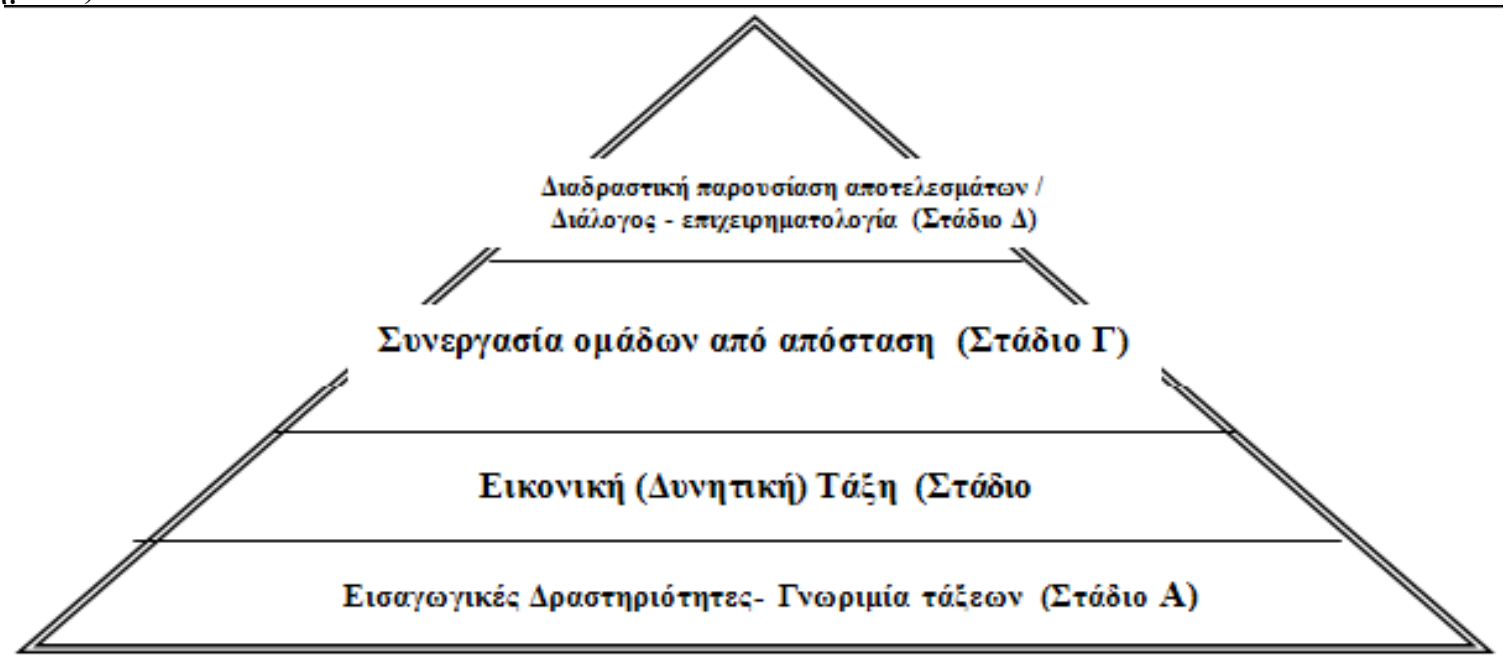

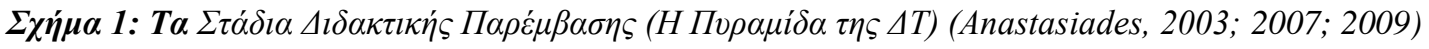

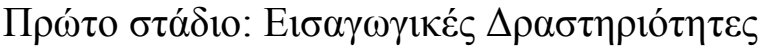

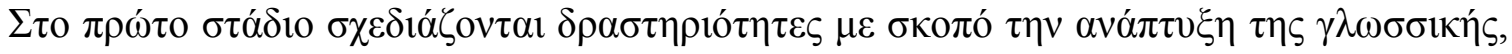

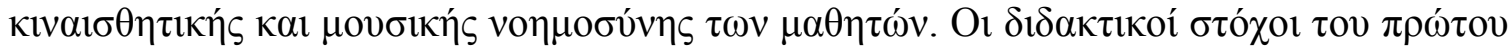

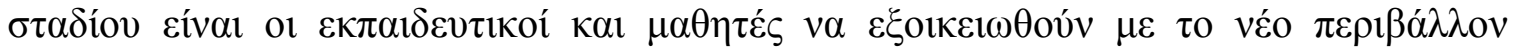




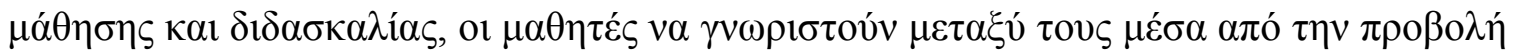

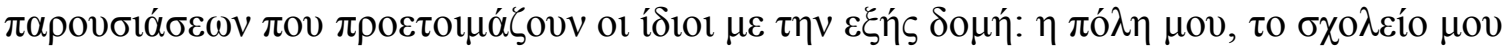

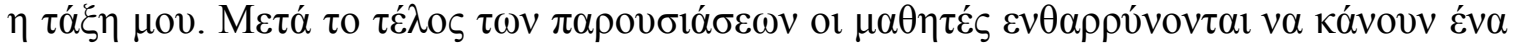

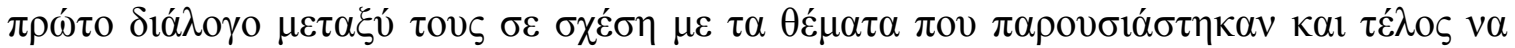

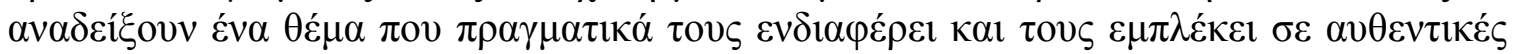

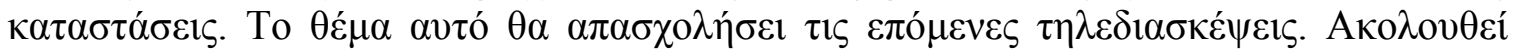

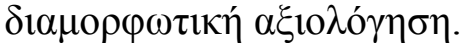

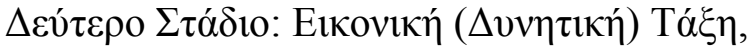

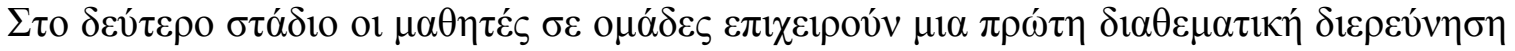

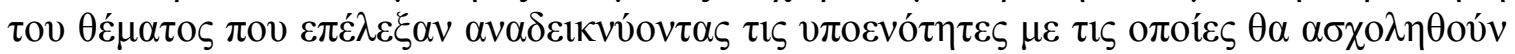

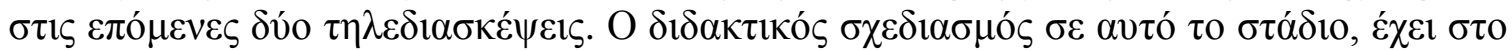

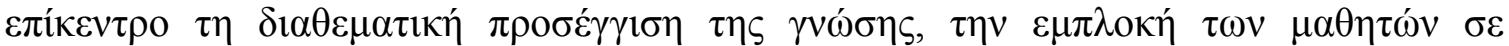

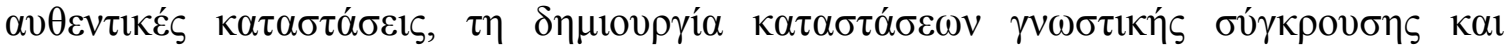

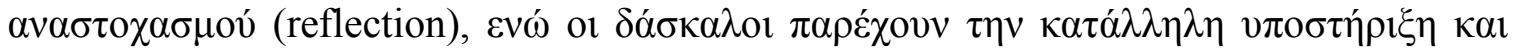

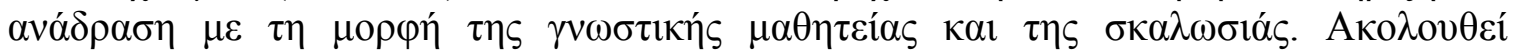

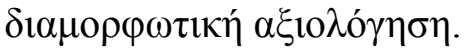

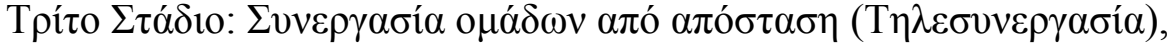

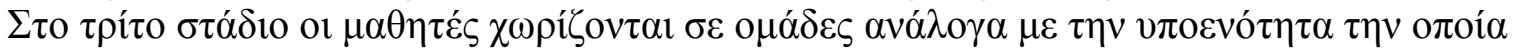

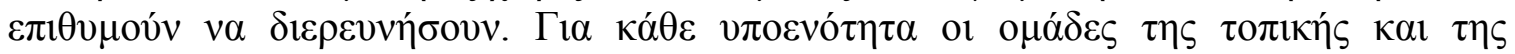

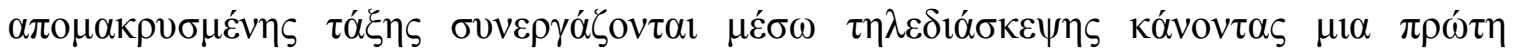

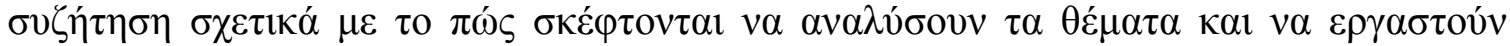

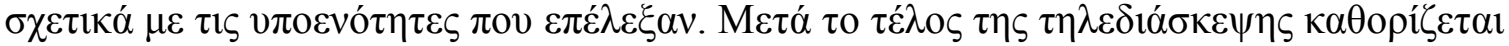

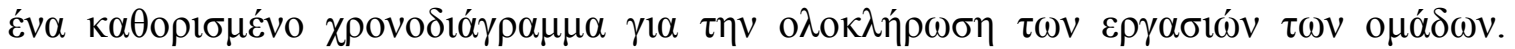

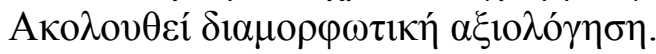

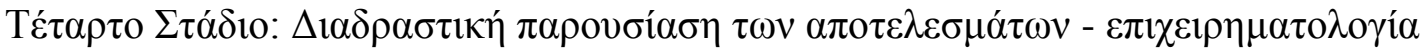

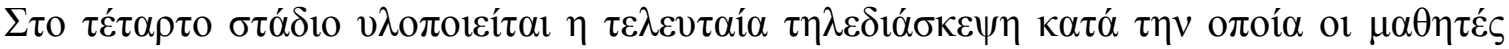

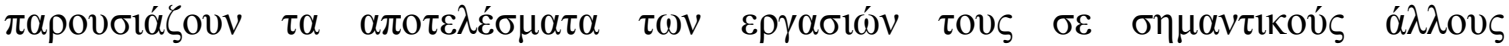

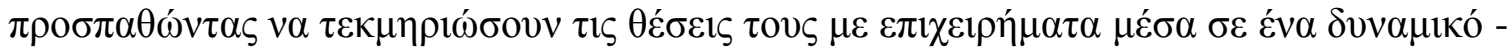

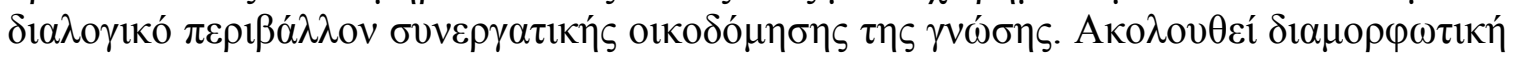

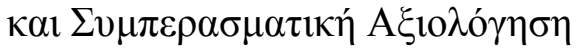

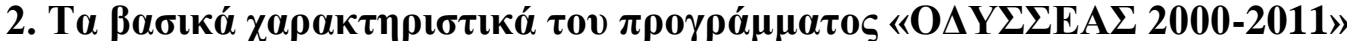

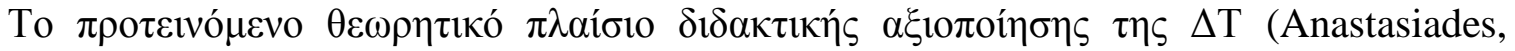

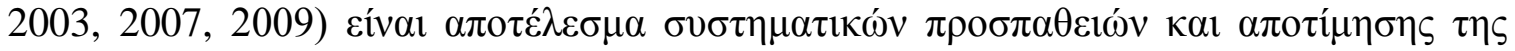

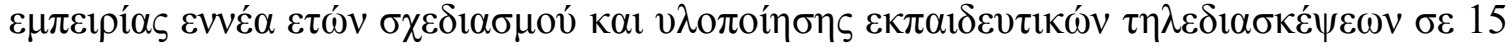

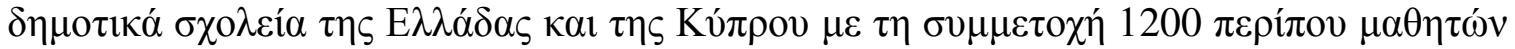

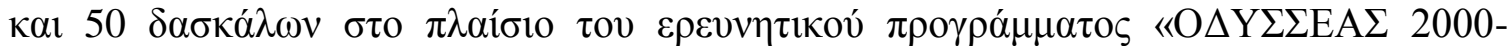
2011».

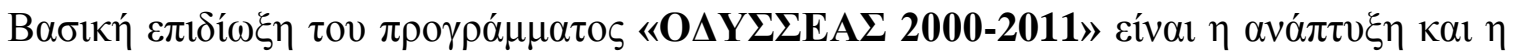

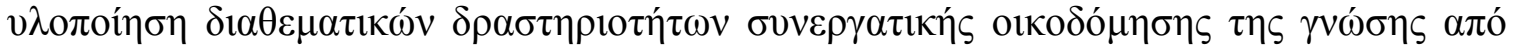

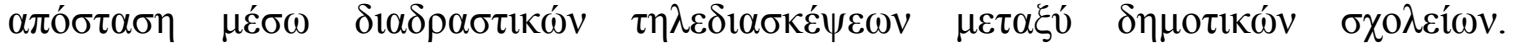
(http://www.edc.uoc.gr/ odysseas/).

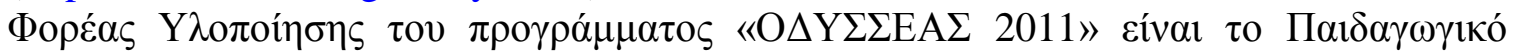

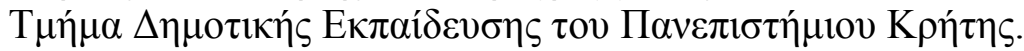

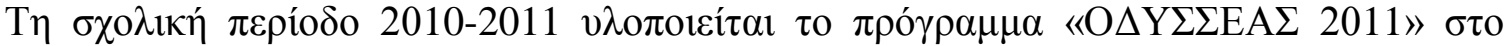

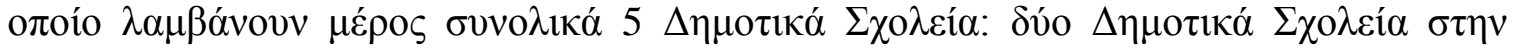




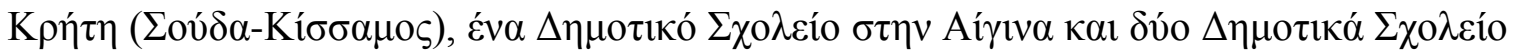

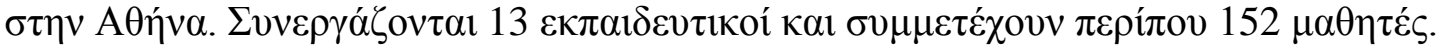

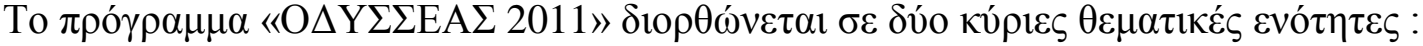

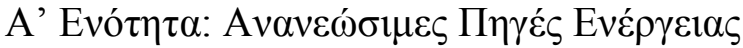

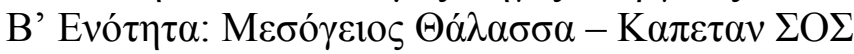

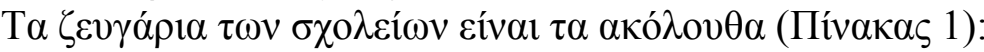

\begin{tabular}{|c|c|}
\hline \multicolumn{2}{|c|}{ 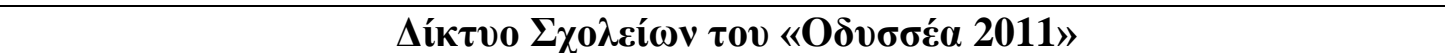 } \\
\hline 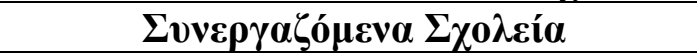 & 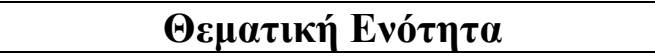 \\
\hline 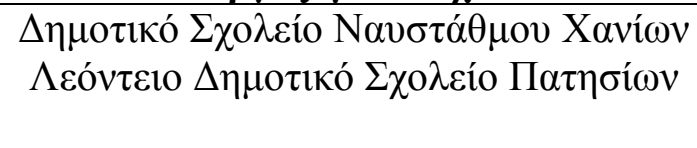 & 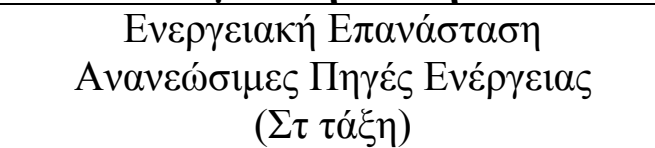 \\
\hline 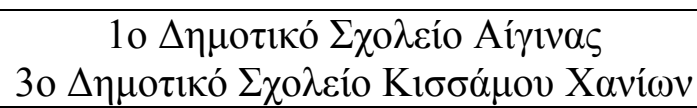 & 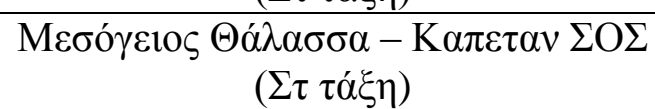 \\
\hline 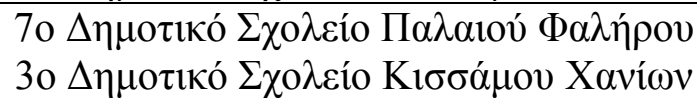 & 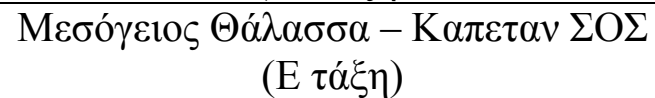 \\
\hline
\end{tabular}

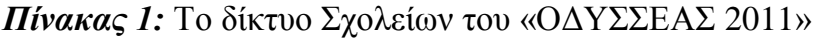

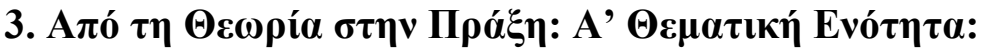

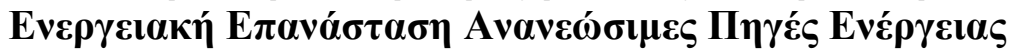

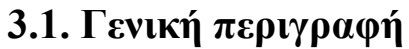

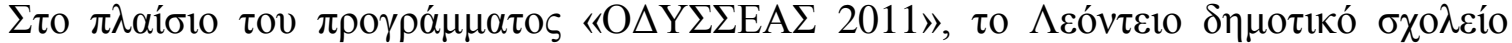

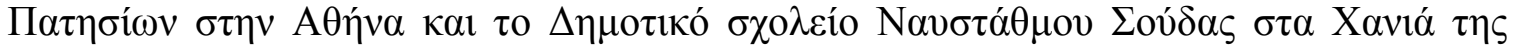

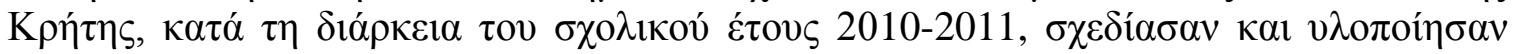

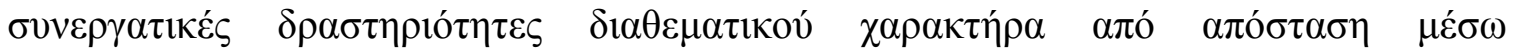

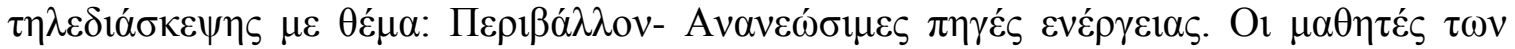

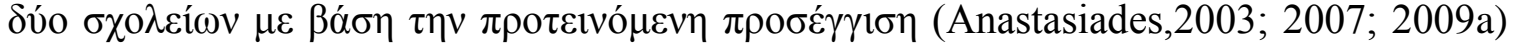

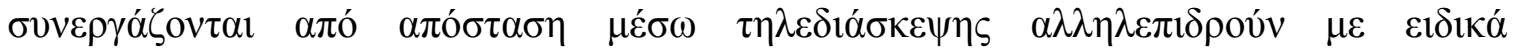

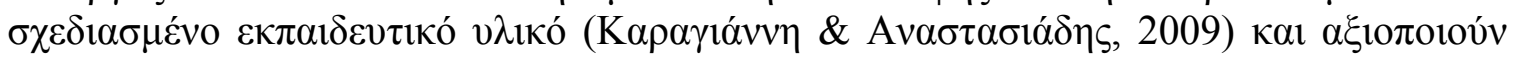

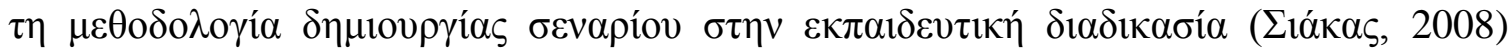

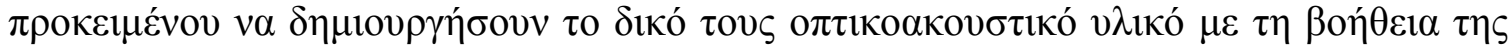

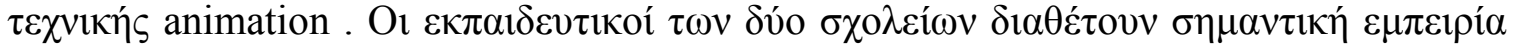

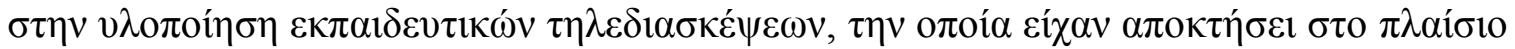

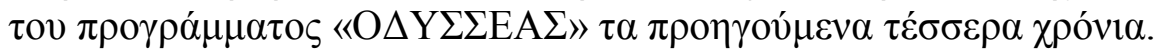

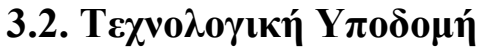

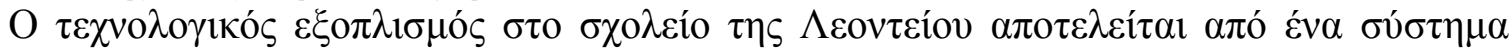

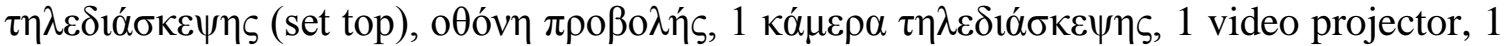

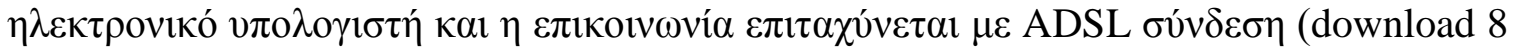

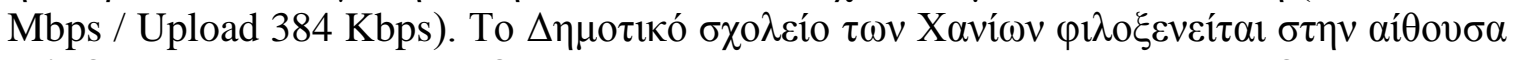

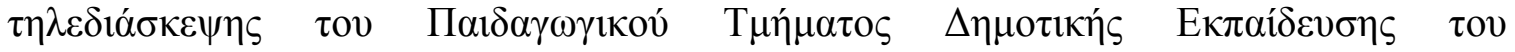

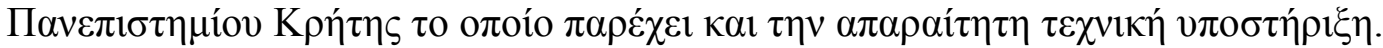

\section{3. $\Sigma \chi \varepsilon \delta 1 \alpha \sigma \mu o ́ \varsigma T \eta \lambda \varepsilon \delta ı \sigma \kappa \varepsilon ́ \psi \varepsilon \omega v$}

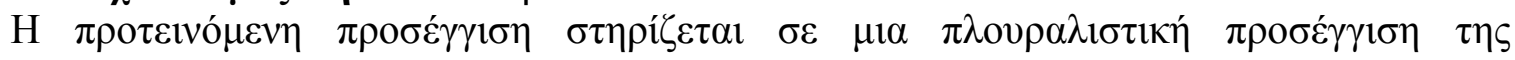

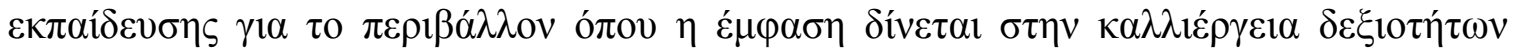

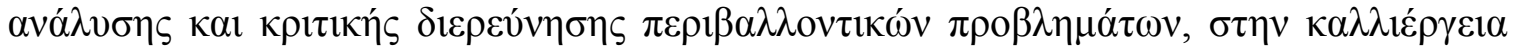


$6^{\text {th }}$ International Conference in Open \& Distance Learning - November 2011, Loutraki, Greece - PROCEEDINGS

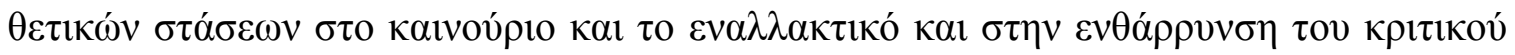

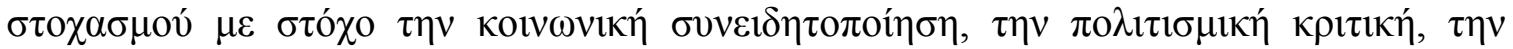

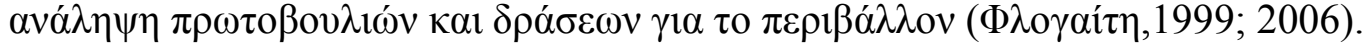

Гı

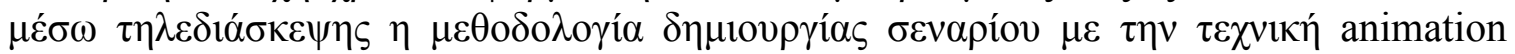

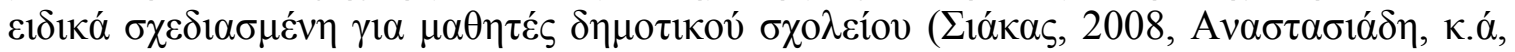

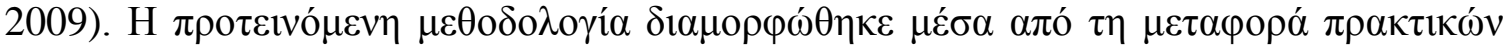

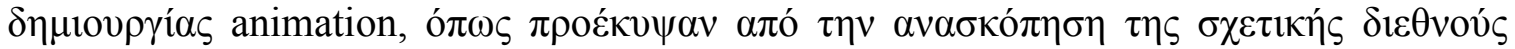
$\beta 1 \beta \lambda$ ıо $\rho \alpha \varphi i ́ \alpha$ (Bordwell, 1985, Bordwell \& Thompson 2004, Branigan, 1992, Halas \&

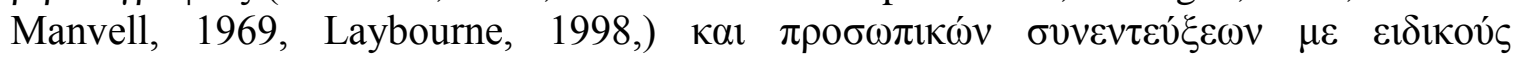

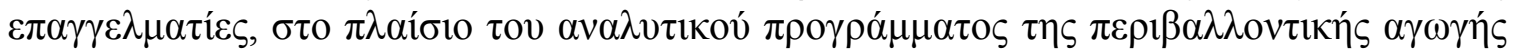

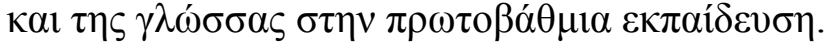

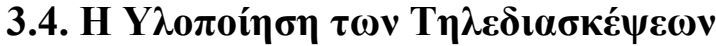

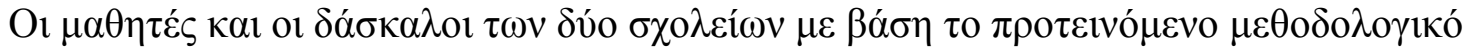

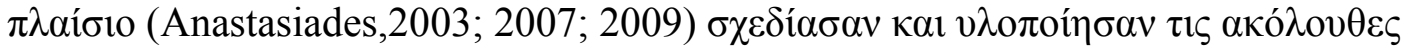

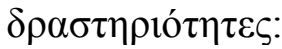

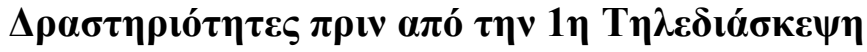

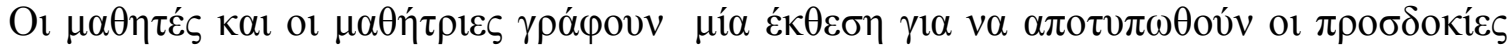

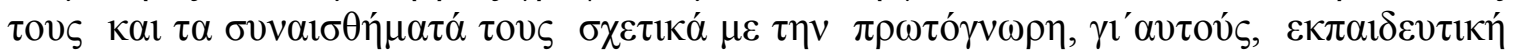

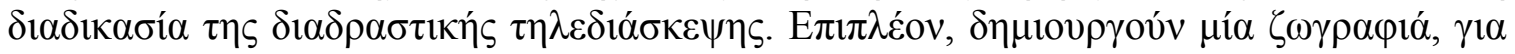

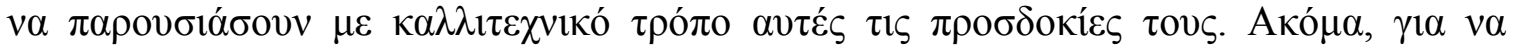

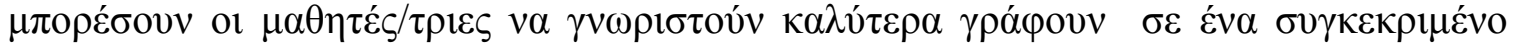

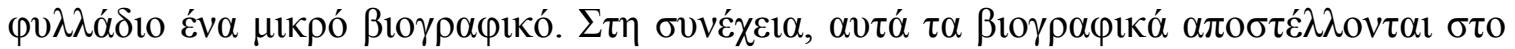

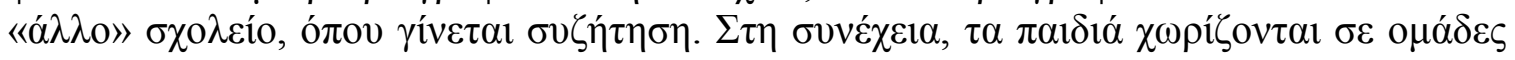

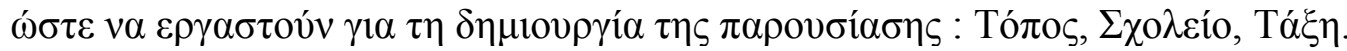

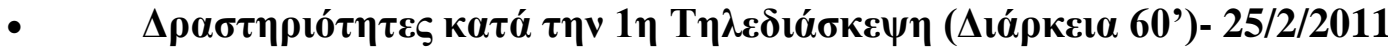

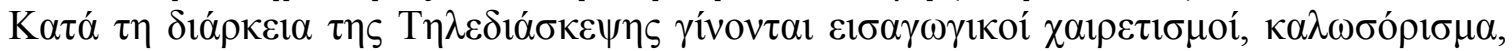

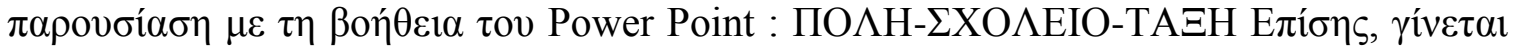

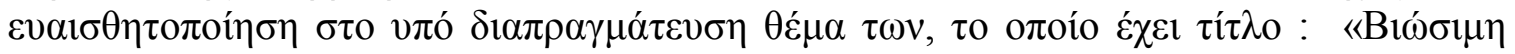

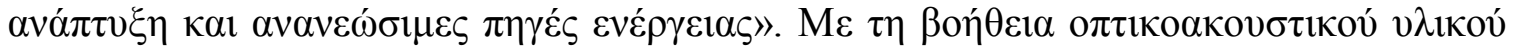

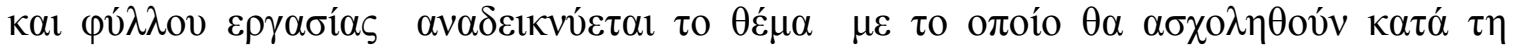

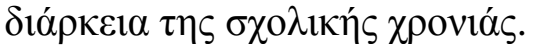

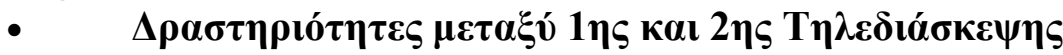

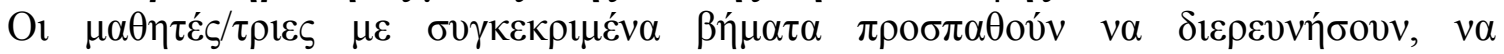

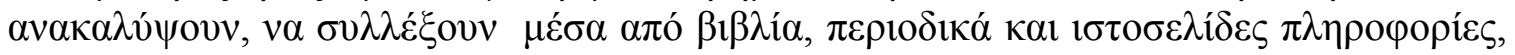

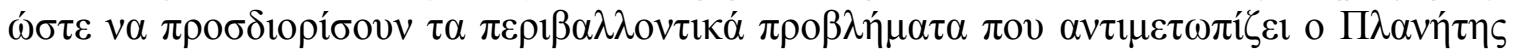

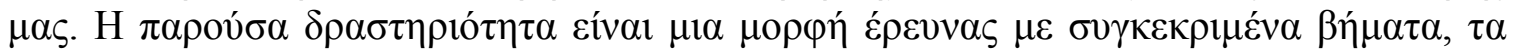

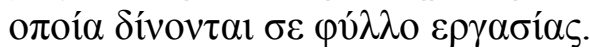

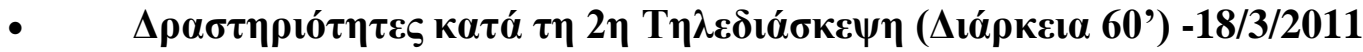

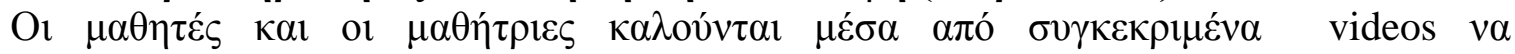

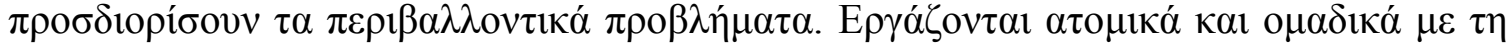

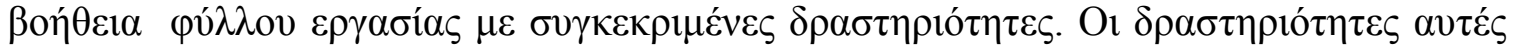

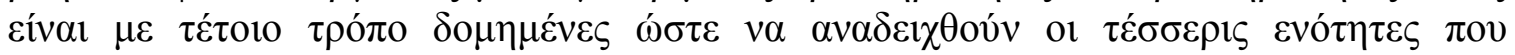

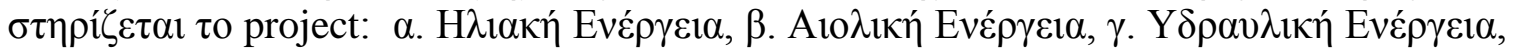

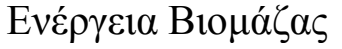

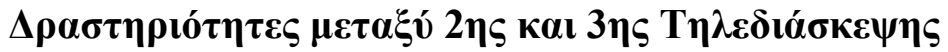




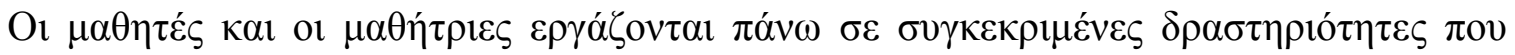

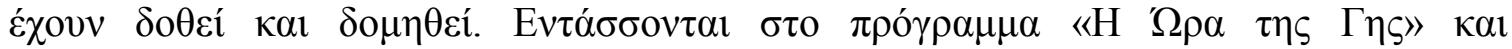

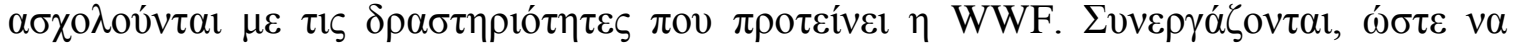

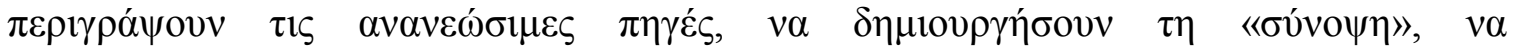

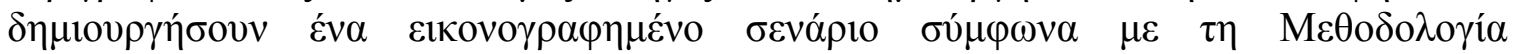

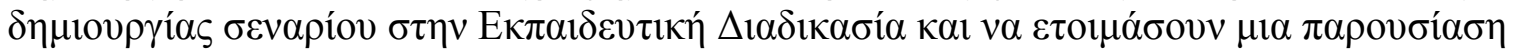

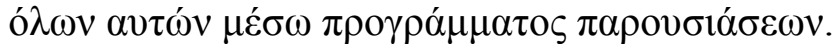

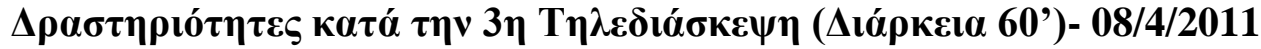

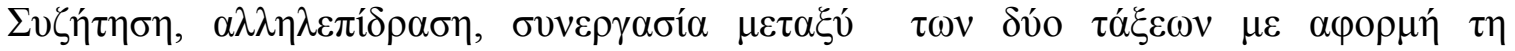

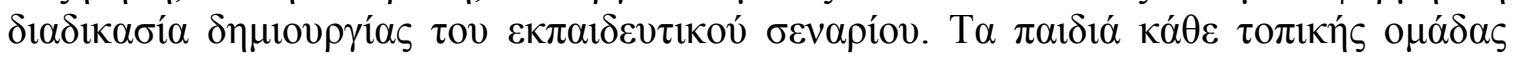

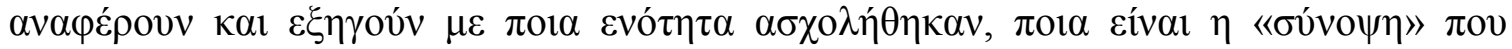

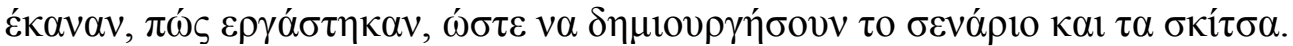

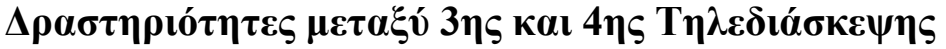

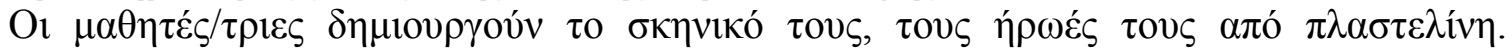

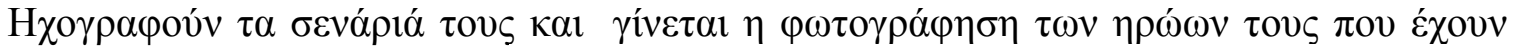

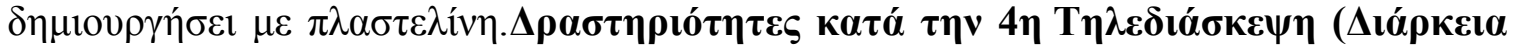
80')- 13/5/2011

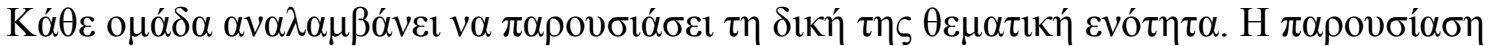

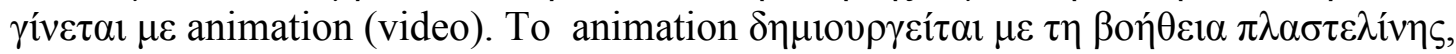

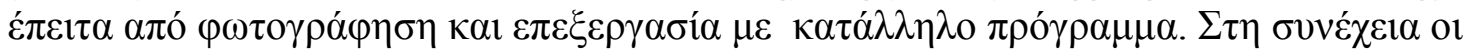

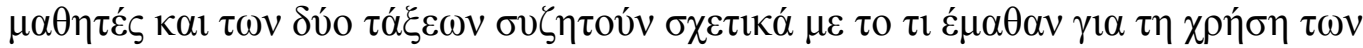

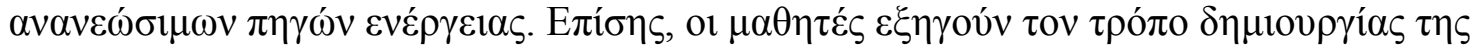

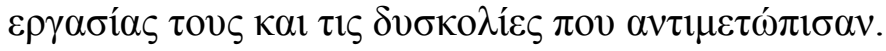

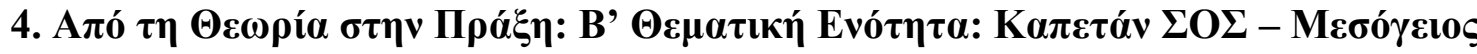

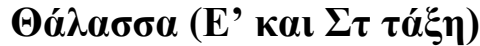

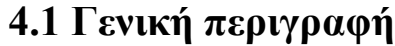

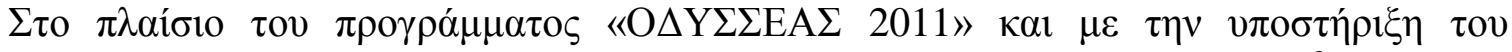

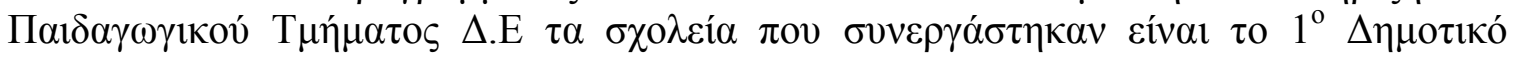

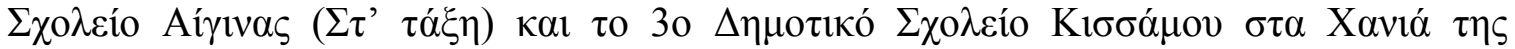

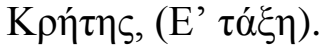

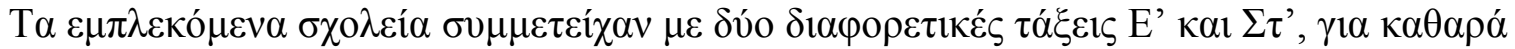

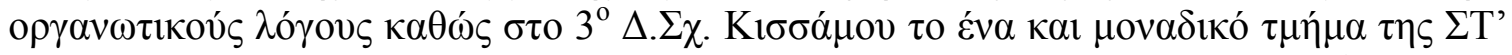

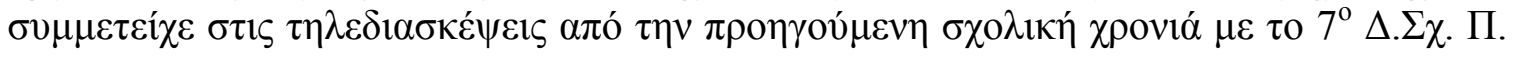

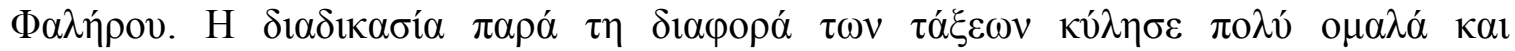

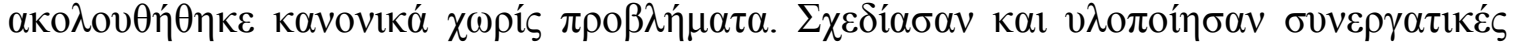

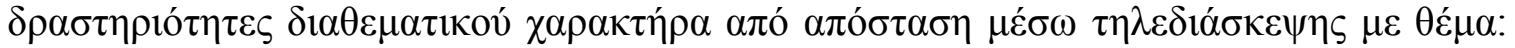

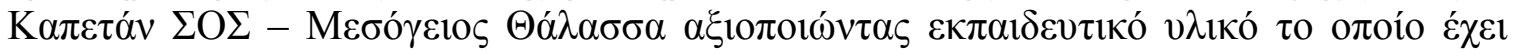

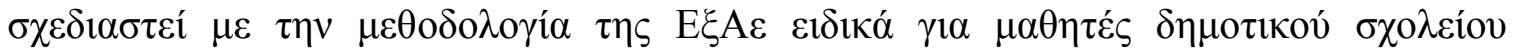
(Mavoúбov, 2007).

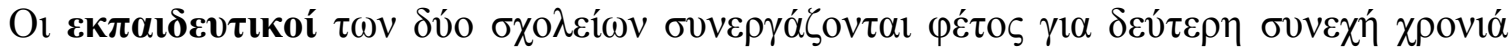

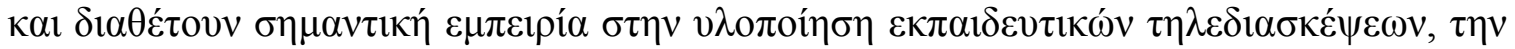

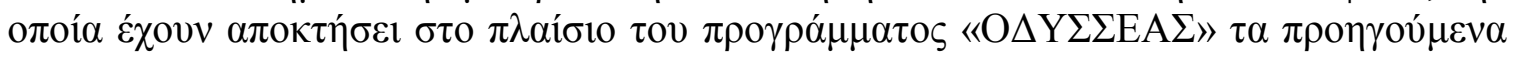
$\chi \rho o ́ v 1 \alpha$. 


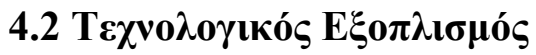

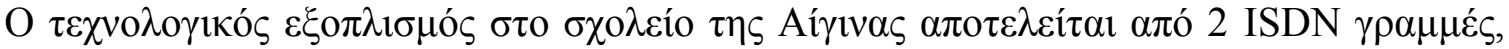

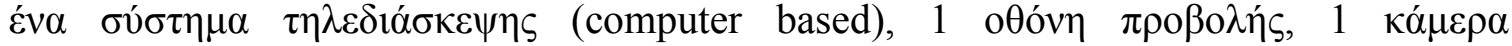

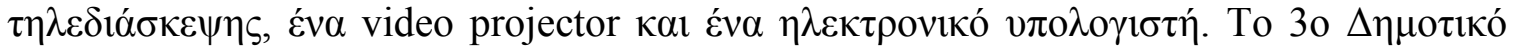

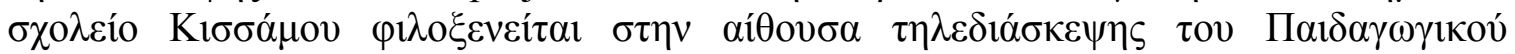

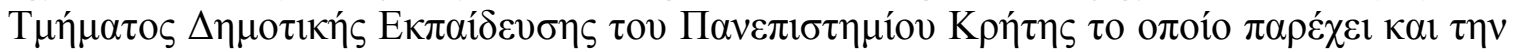

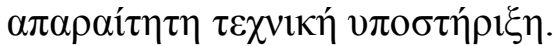

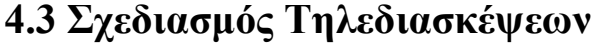

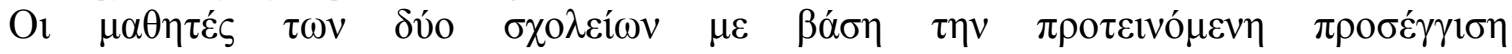

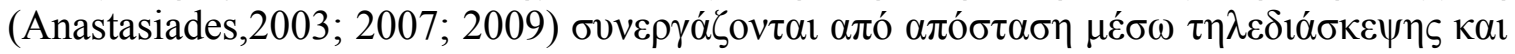
$\alpha \xi$ lo

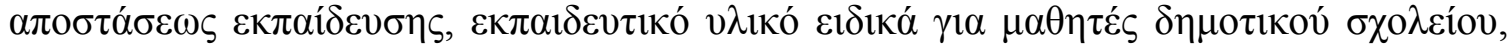

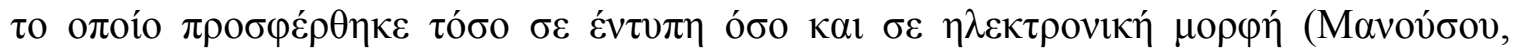

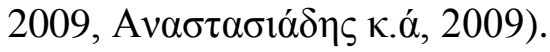

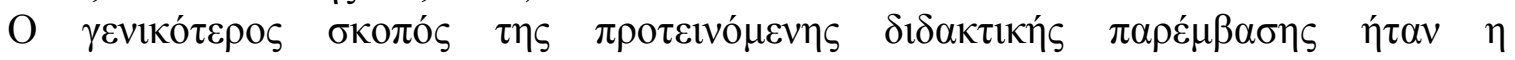

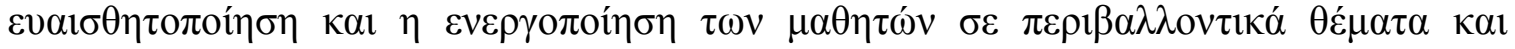

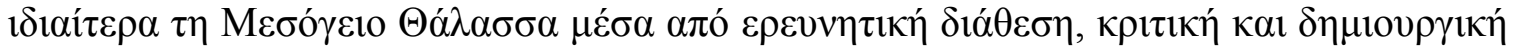

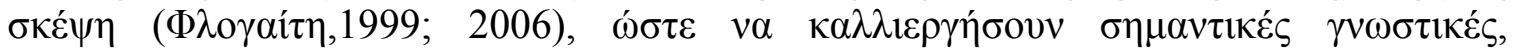

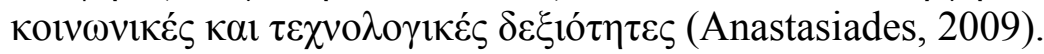

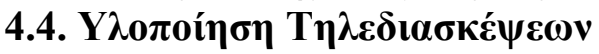

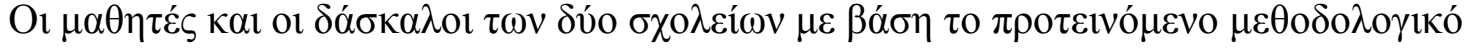

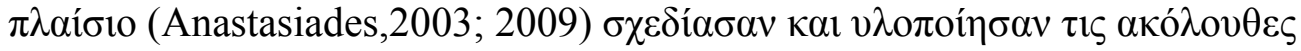
$\delta \rho \alpha \sigma \tau \eta \rho \tau^{\tau} \eta \tau \varepsilon \zeta$.

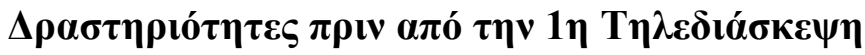

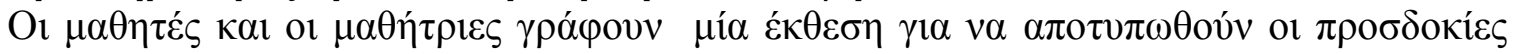

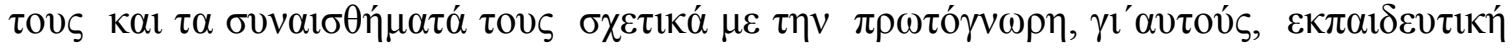

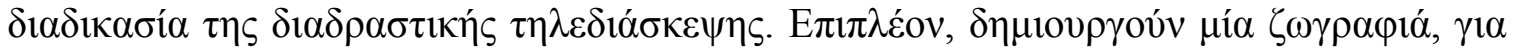

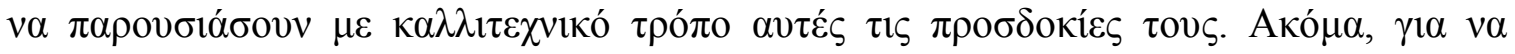

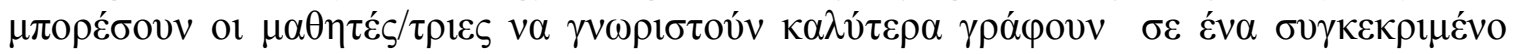

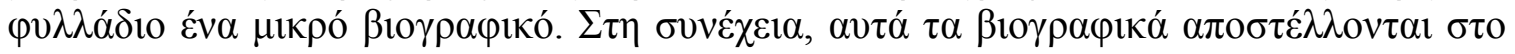

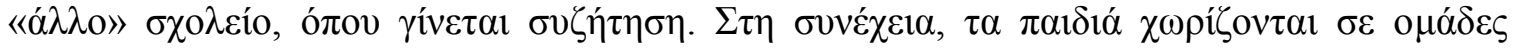

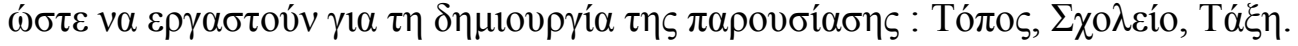

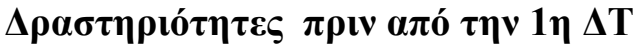

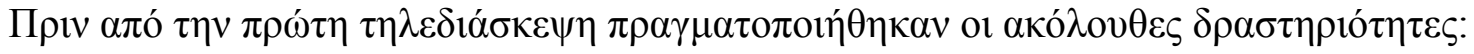

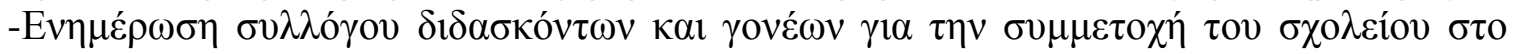

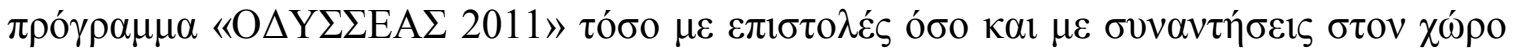

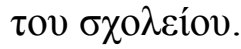

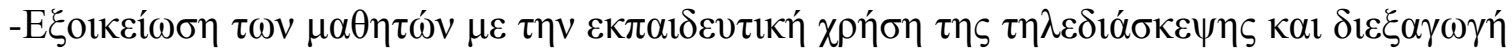

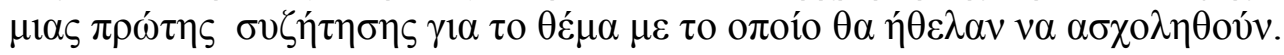

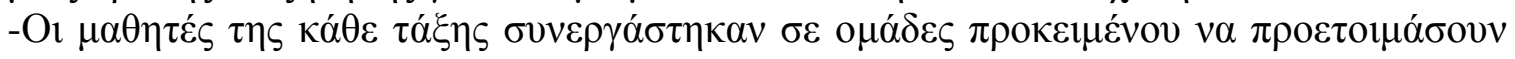

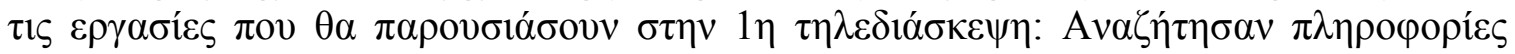

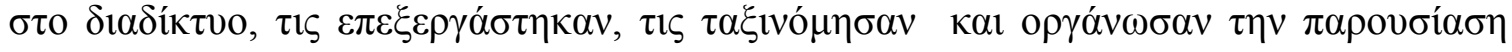
$\tau$ ¿ov $\sigma \varepsilon$ power point.

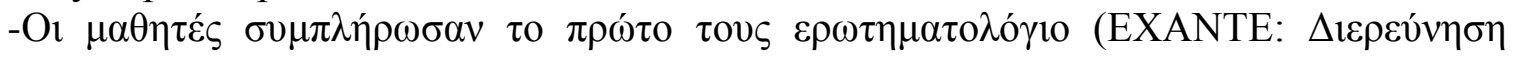

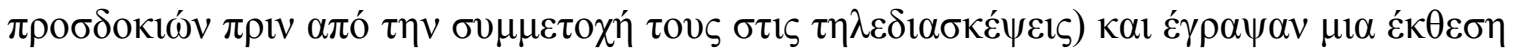


$6^{\text {th }}$ International Conference in Open \& Distance Learning - November 2011, Loutraki, Greece - PROCEEDINGS

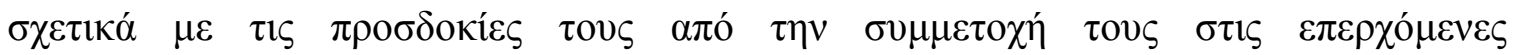
$\tau \eta \lambda \varepsilon \delta 1 \alpha \sigma \kappa \varepsilon ́ \psi \varepsilon 1 \zeta$

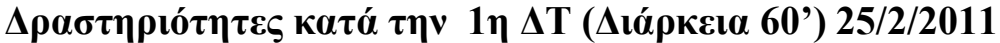

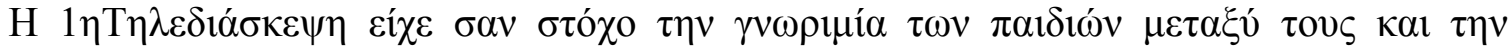

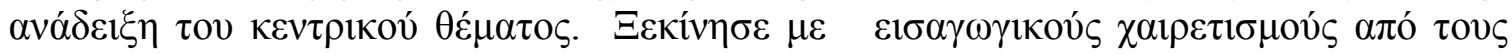

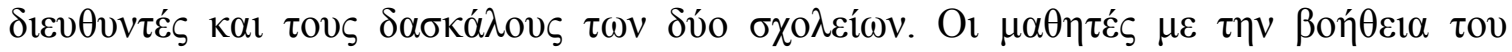

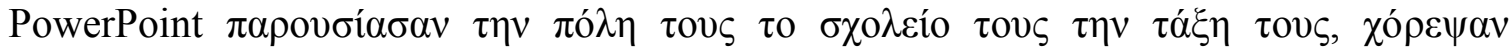

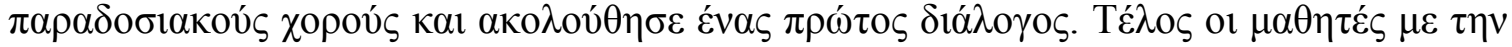

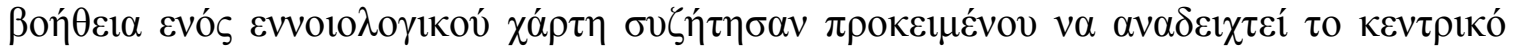

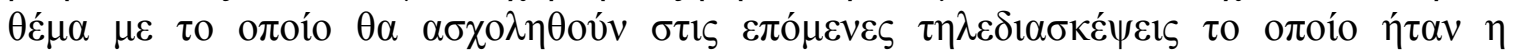

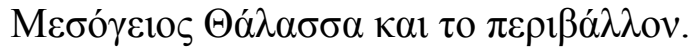

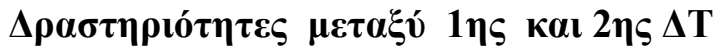

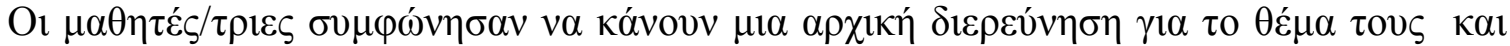

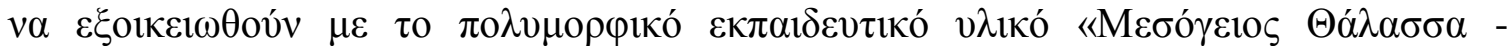

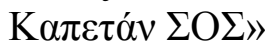

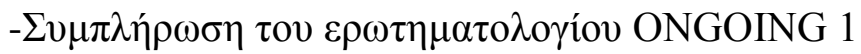

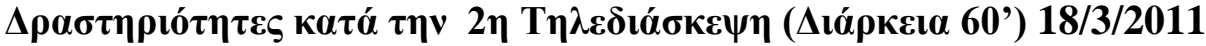

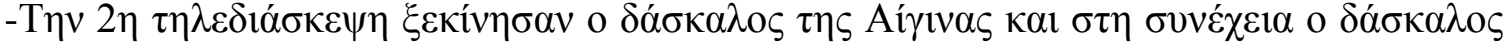

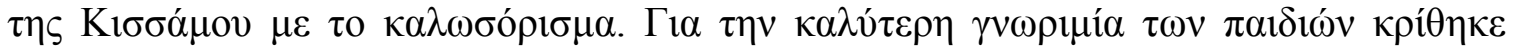

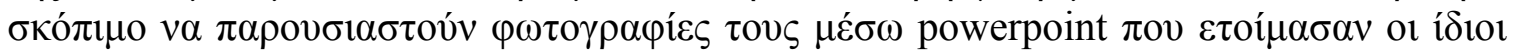
ol $\mu \alpha \theta \eta \tau \dot{\varepsilon} \varsigma$

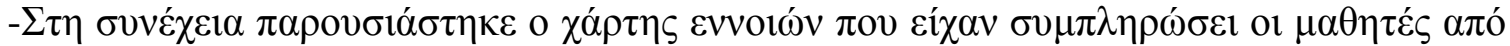

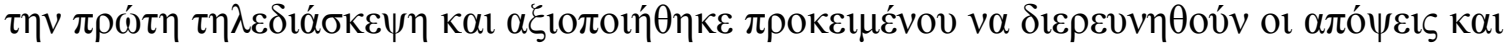

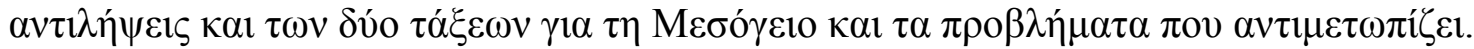

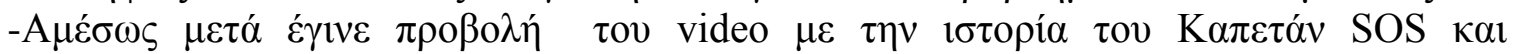

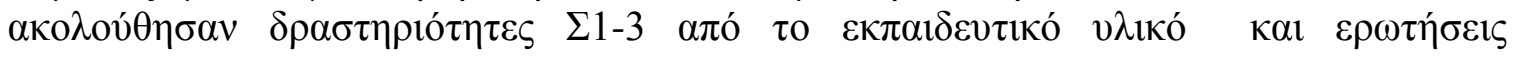

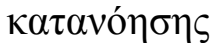

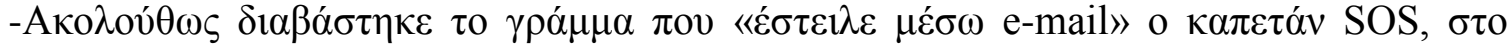

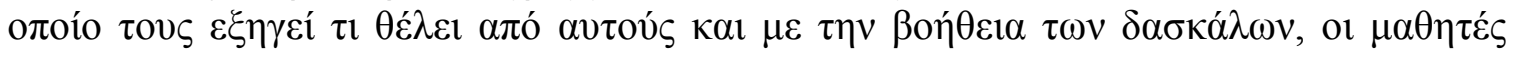

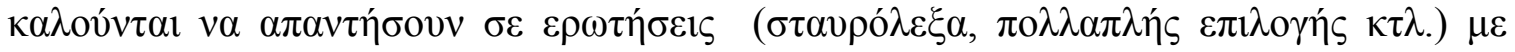

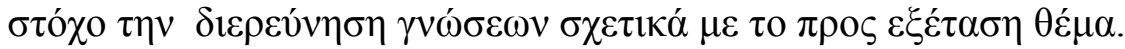

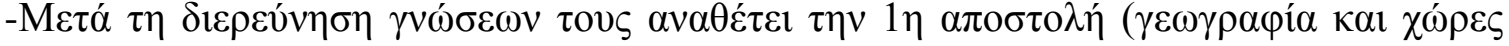

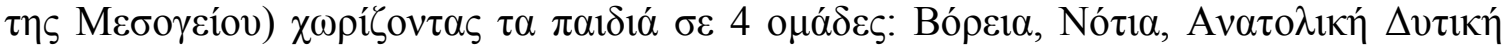

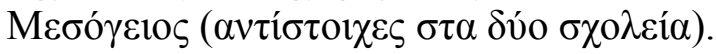

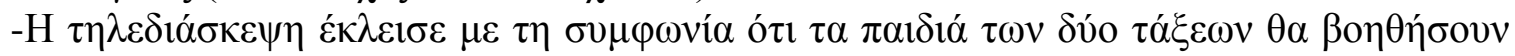

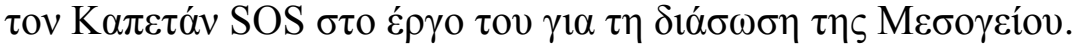

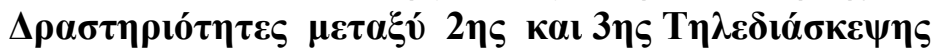

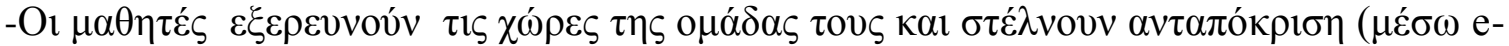

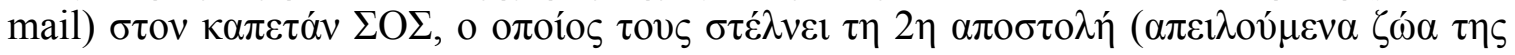

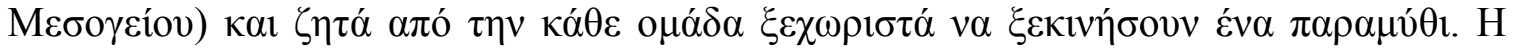

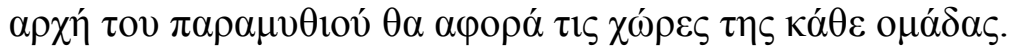

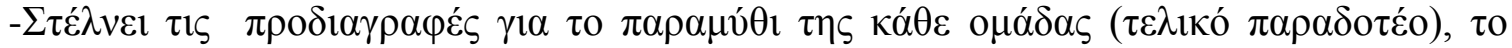

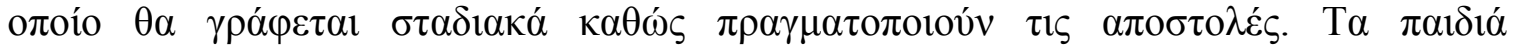

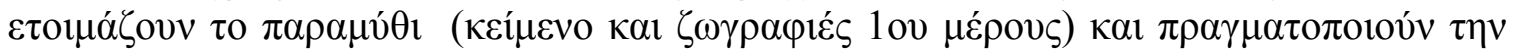

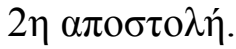




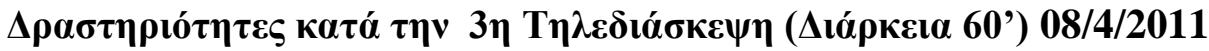

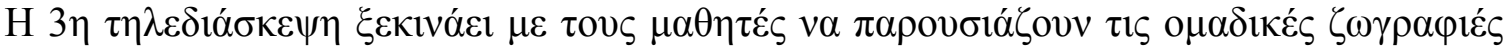
$\tau \mathrm{O} v \mathrm{~K} \alpha \pi \varepsilon \tau \alpha \dot{v} v \mathrm{O} \Sigma$.

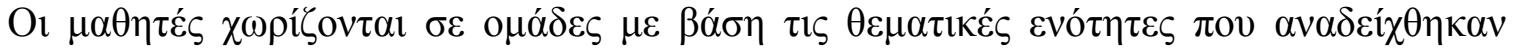

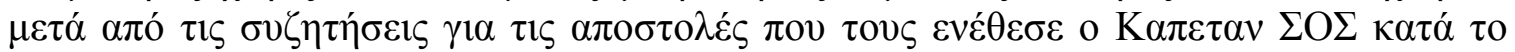

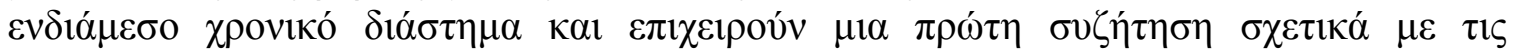

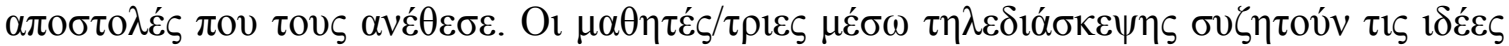

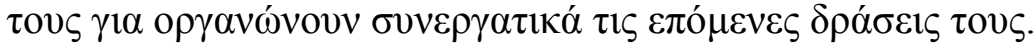

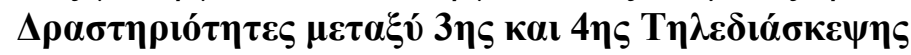

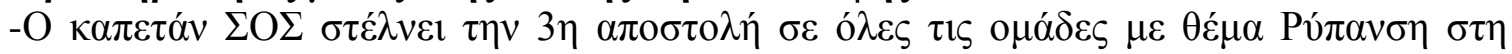

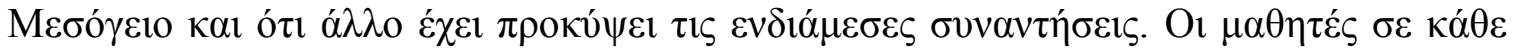

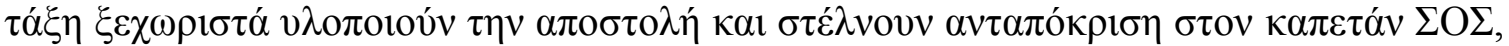

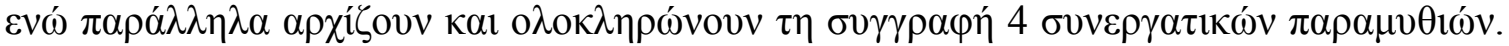

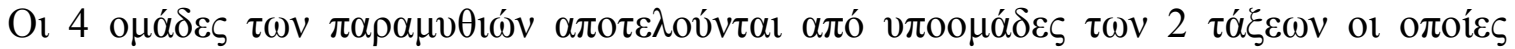

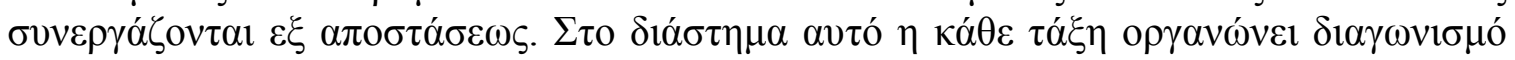

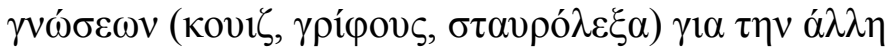

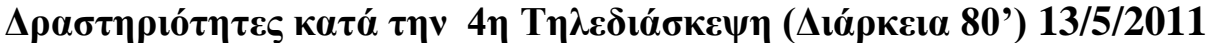

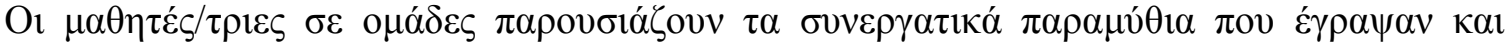

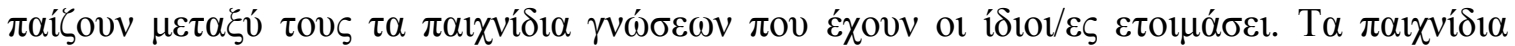

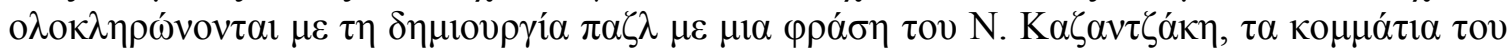

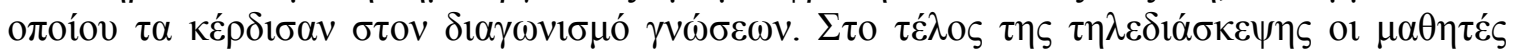

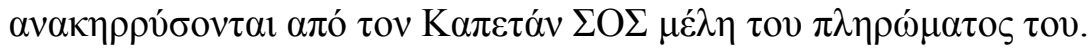

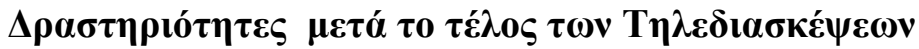

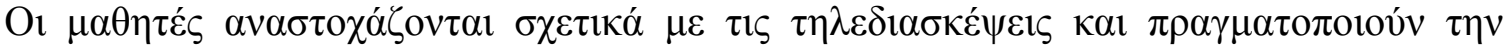

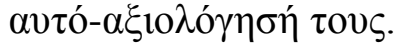

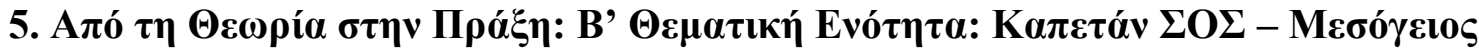

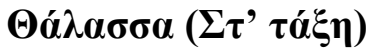

5.1 Геvıкท́ $\pi \varepsilon \rho \iota \gamma \rho \alpha \varphi ́$

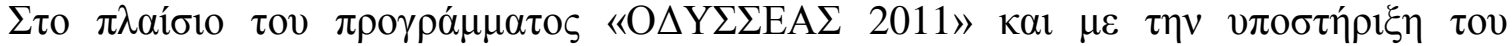

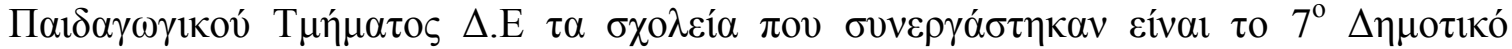

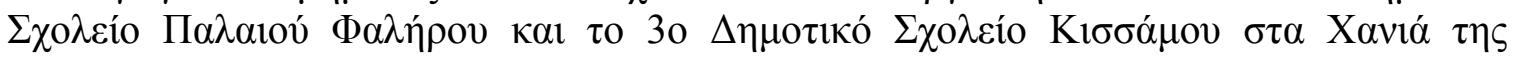

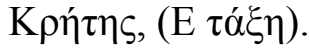

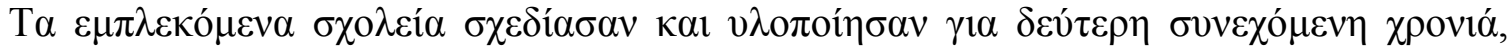

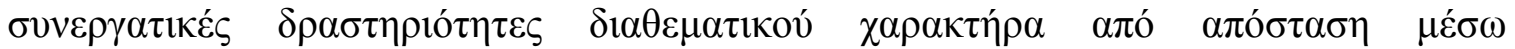

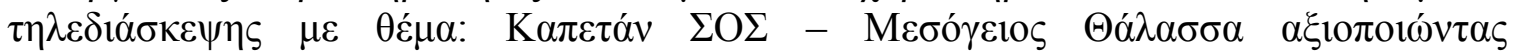

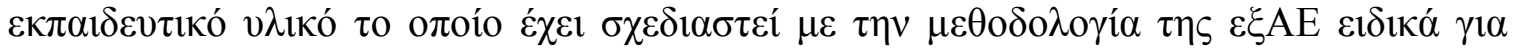

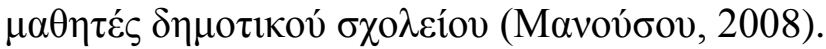

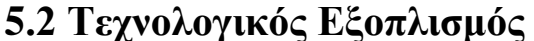

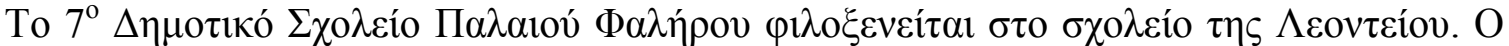

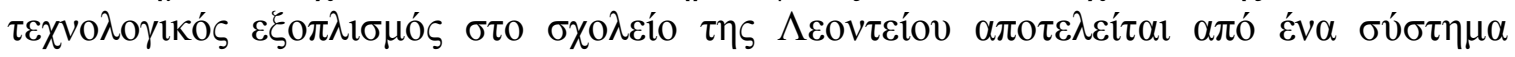

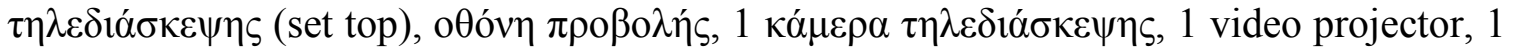

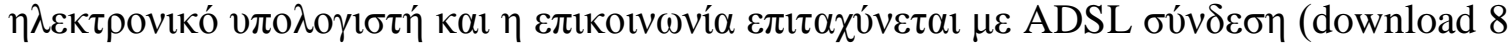

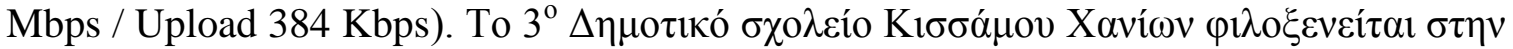

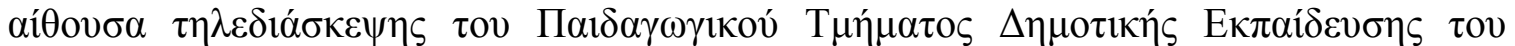

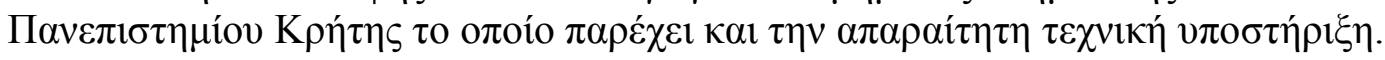




\section{$5.3 \Sigma \chi \varepsilon \delta\llcorner\alpha \sigma \mu o ́ \varsigma \mathrm{T} \eta \lambda \varepsilon \delta\llcorner\alpha \sigma \kappa \varepsilon ́ \psi \varepsilon \omega v$}

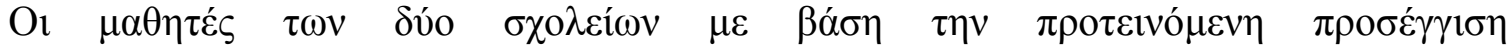

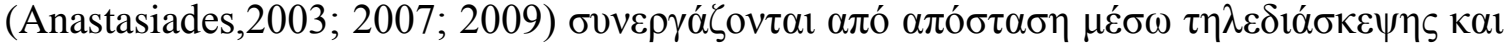

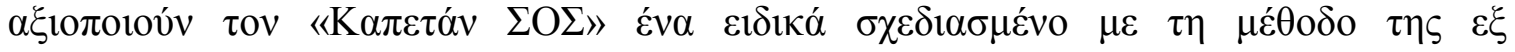

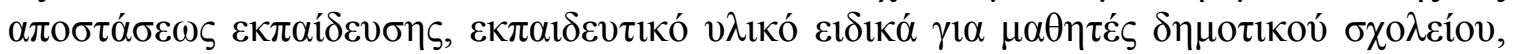

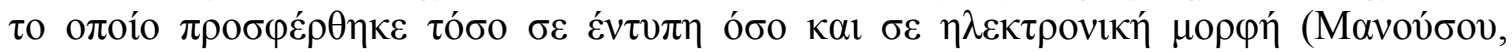
2009).

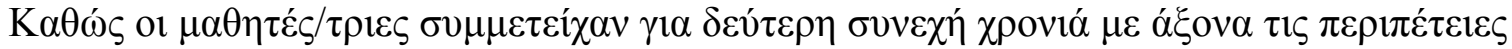

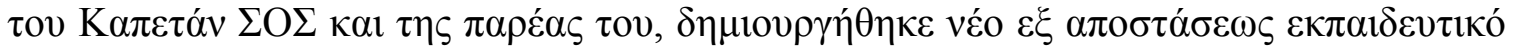

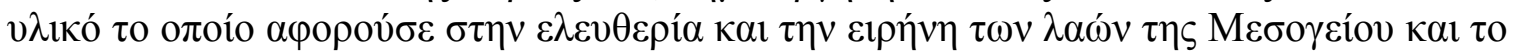

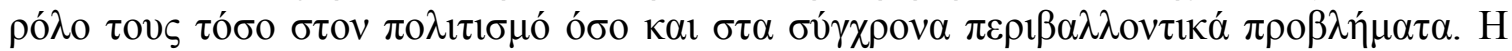

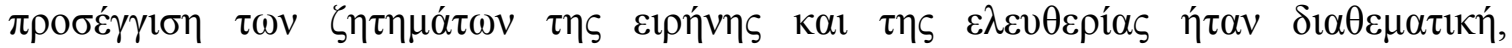

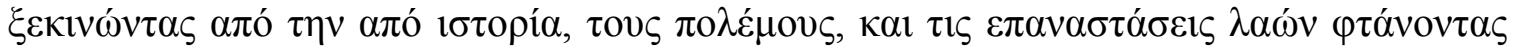

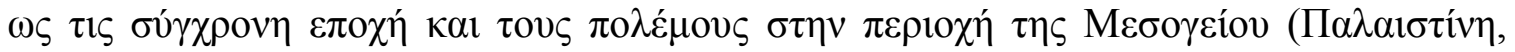

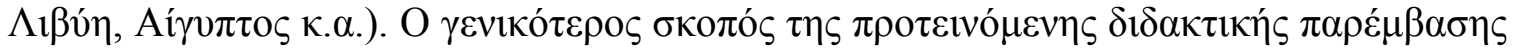

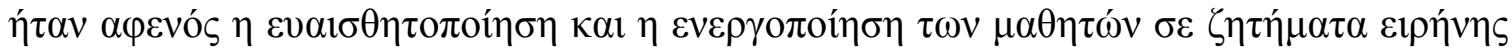

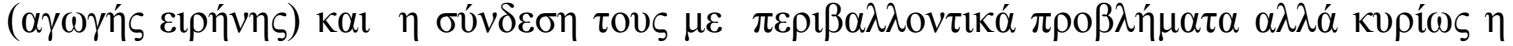

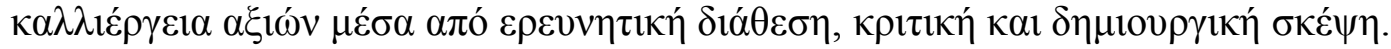

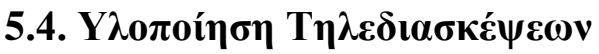

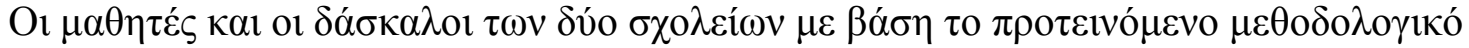

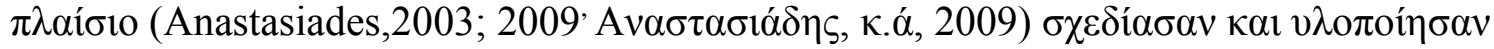
$\tau 1 \varsigma \alpha \kappa o ́ \lambda \sigma v \theta \varepsilon \varsigma \delta \rho \alpha \sigma \tau \eta \rho \imath o ́ \tau \eta \tau \varepsilon \varsigma$.

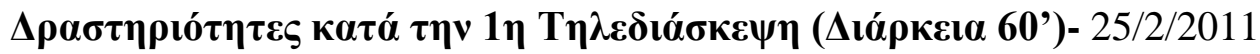

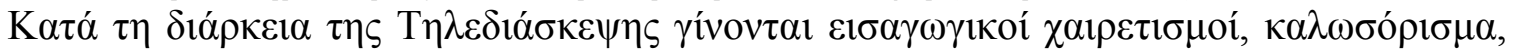

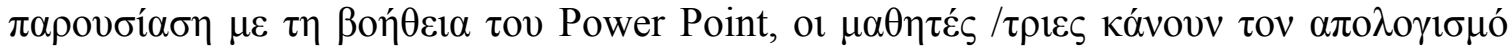

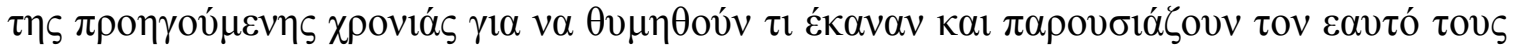

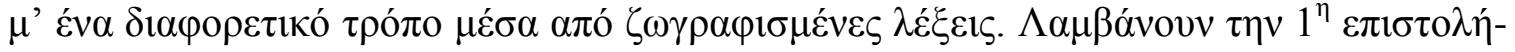

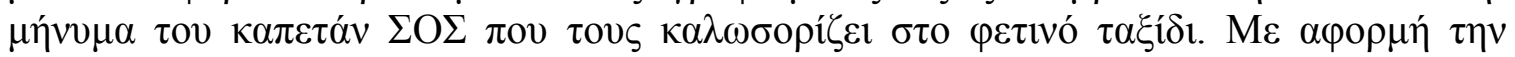

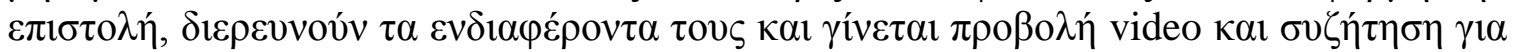

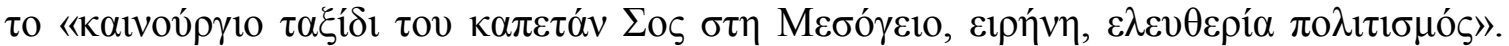

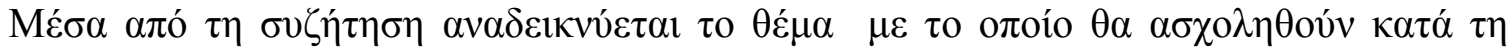

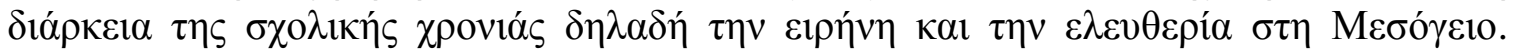
(2011).

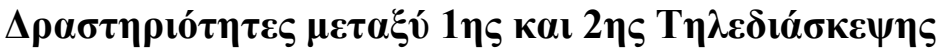

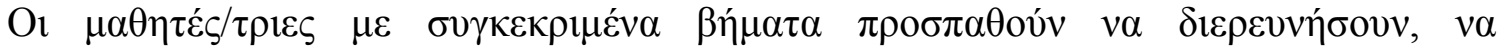

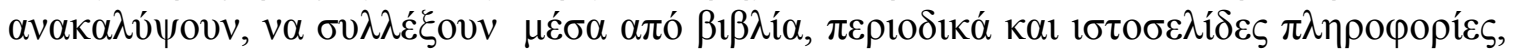

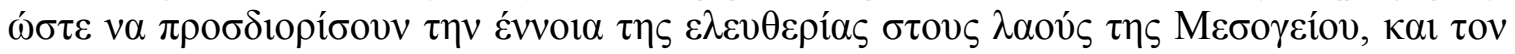

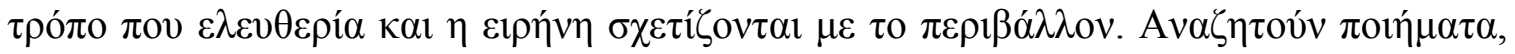

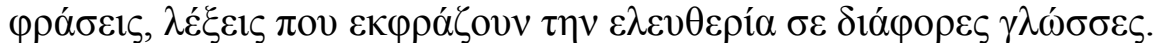

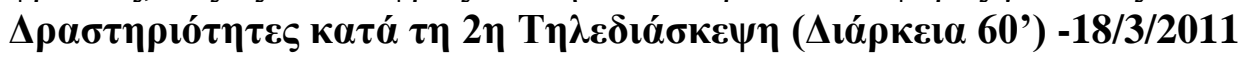

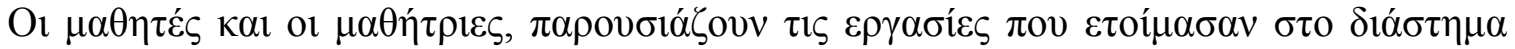

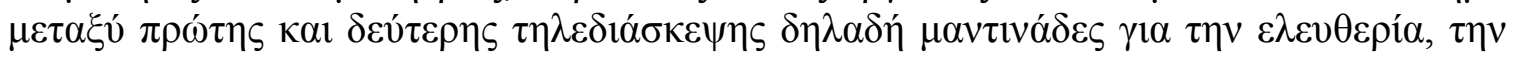

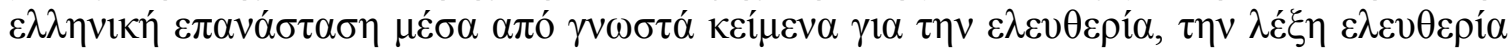

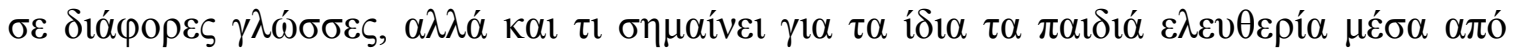


$6^{\text {th }}$ International Conference in Open \& Distance Learning - November 2011, Loutraki, Greece - PROCEEDINGS

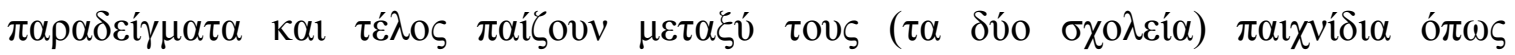
$\alpha \kappa \rho о \sigma \tau \imath \chi i ́ \delta \varepsilon \varsigma \gamma \jmath \alpha \tau \eta \nu \varepsilon \lambda \varepsilon v \theta \varepsilon \rho i ́ \alpha$

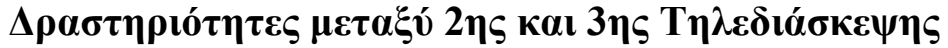

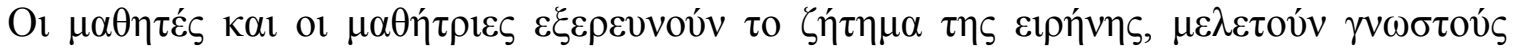

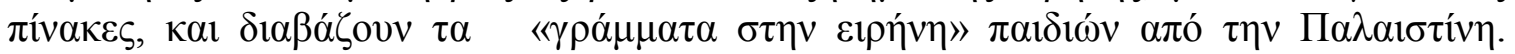

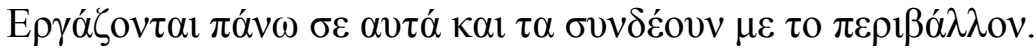

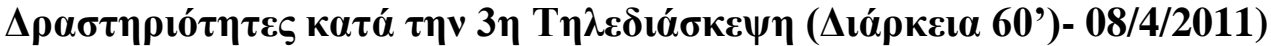

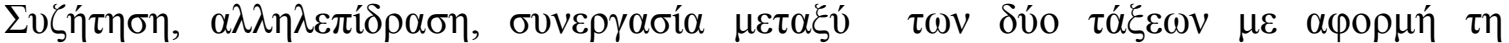

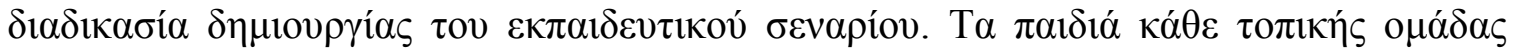

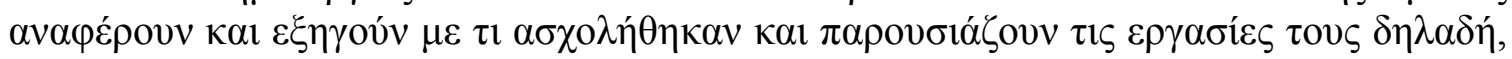

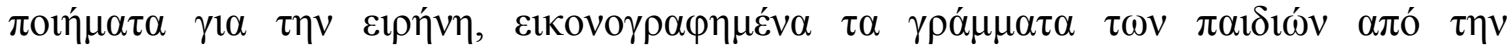

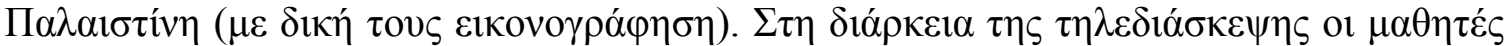

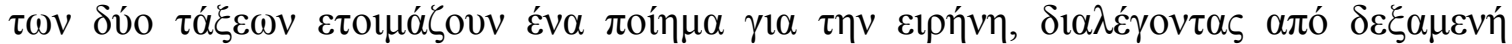

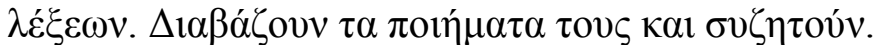

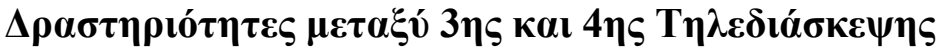

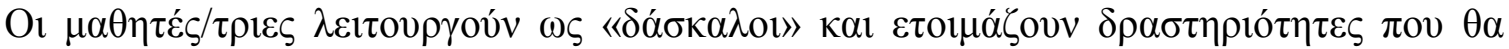

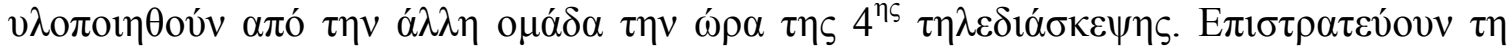

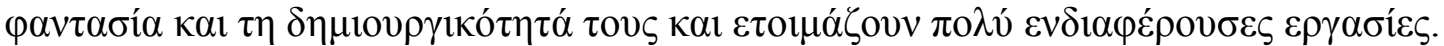

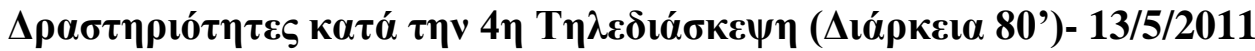

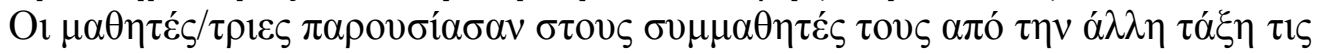

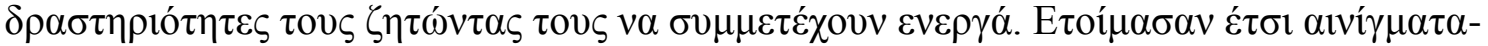

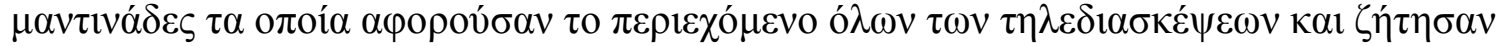

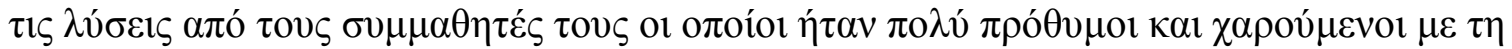

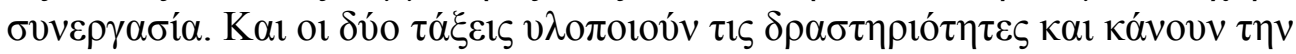

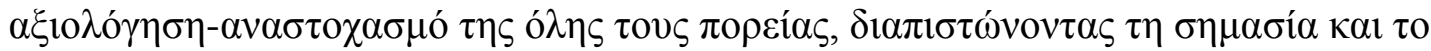

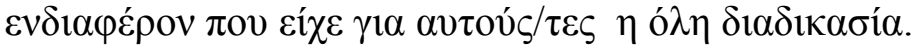

\section{$\Sigma v \mu \pi \varepsilon \rho \alpha ́ \sigma \mu \alpha \tau \alpha$}

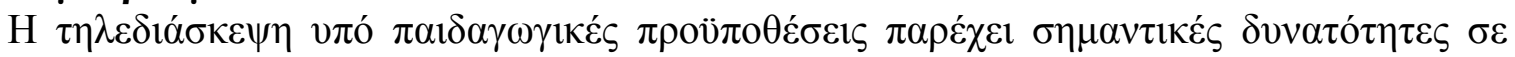

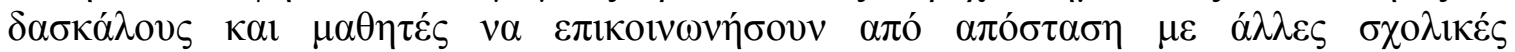

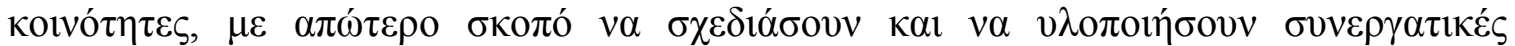

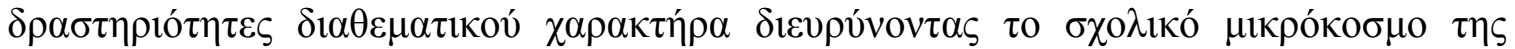

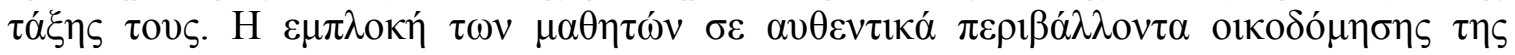

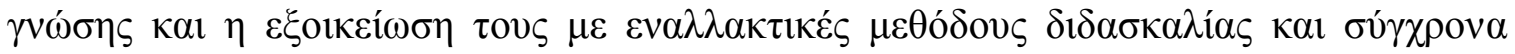

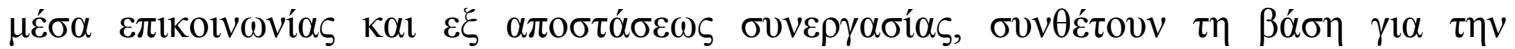

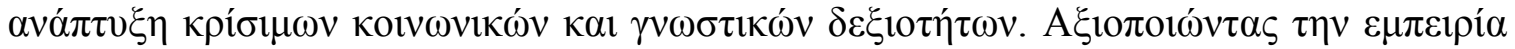

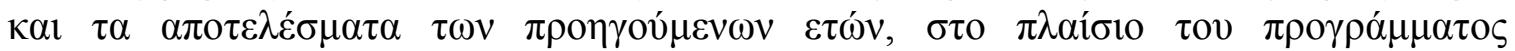

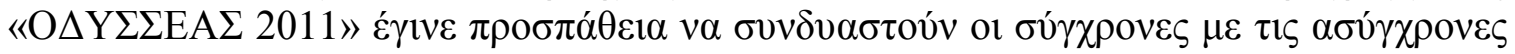

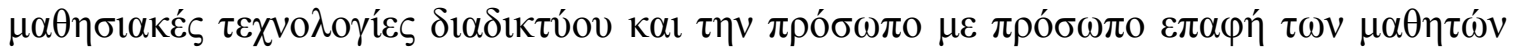

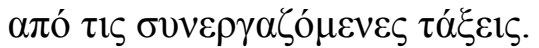

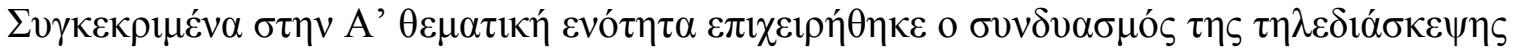

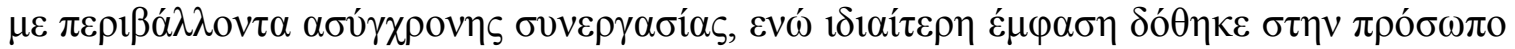

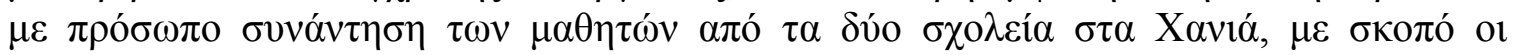

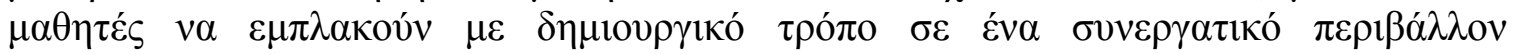

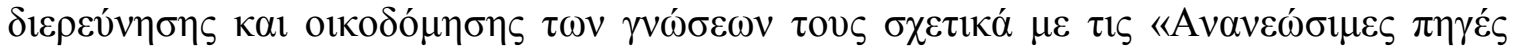

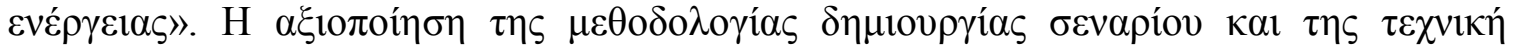




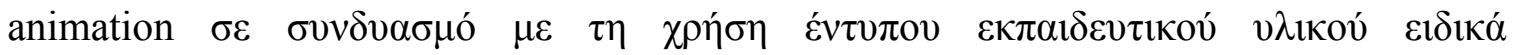

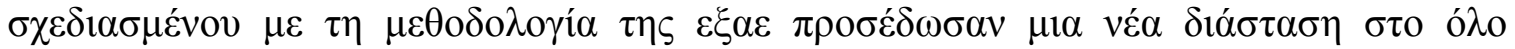
$\varepsilon \gamma \chi \varepsilon i ́ p \eta \mu \alpha$.

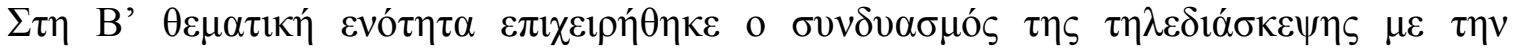

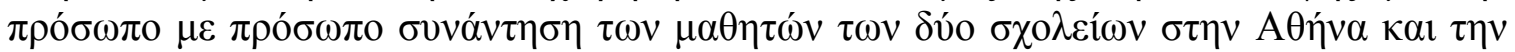

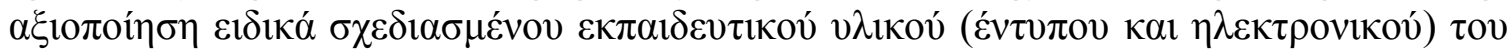

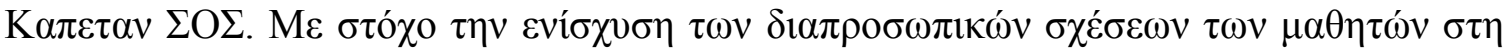

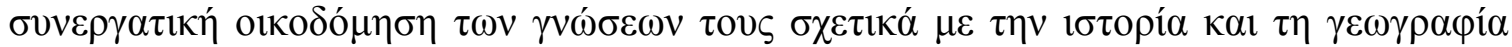

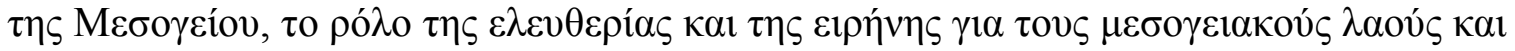

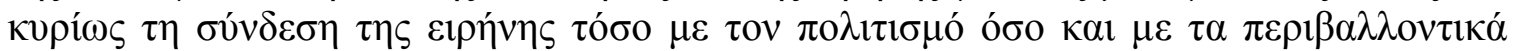

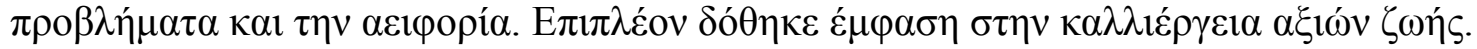

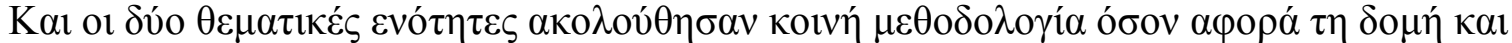

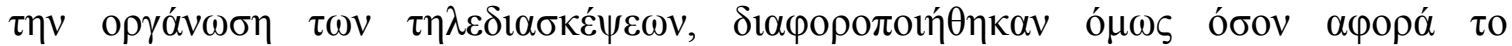

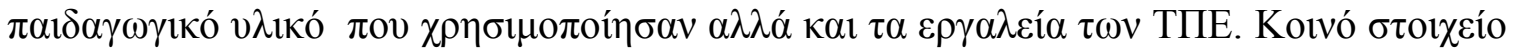

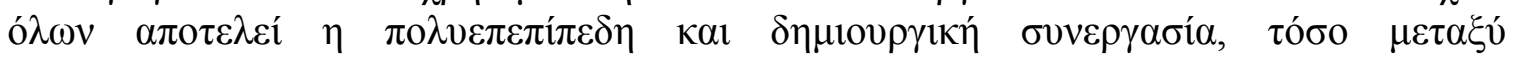

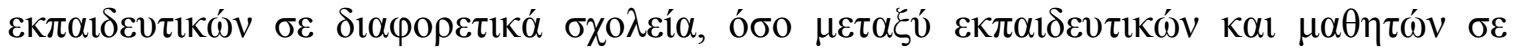

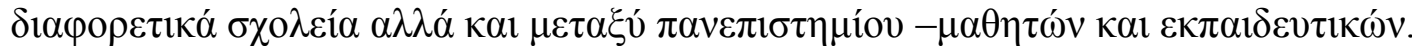

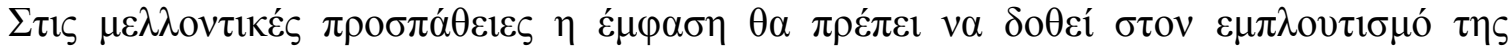

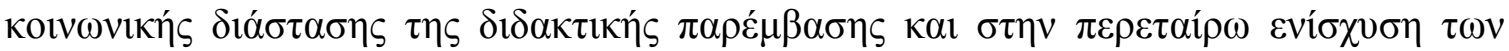

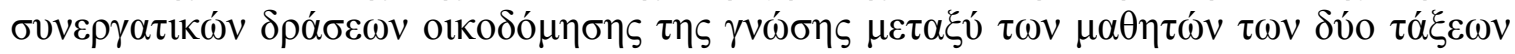

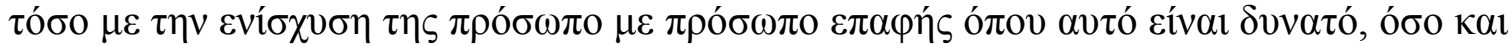

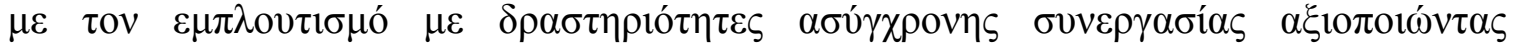

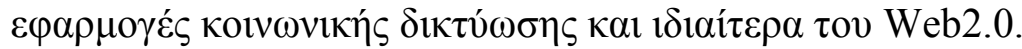

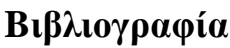

\section{$\Xi \varepsilon v o ́ \gamma \lambda \omega \sigma \sigma \eta$}

Alexander, W., Higgison, C., \& Mogey, N. (Eds.). (1999).Videoconferencing in teaching and learning: Case studies. LTDI and TALiSMAN, Institute of Computer-Based Learning. Heriot-Watt University Edinburgh, SCT. Retrieved on 4 June, 2004, from www.icbl.hw.ac.uk/ltdi

Ally, M. (2004). Foundations of educational theory for online learning. In T. Anderson and F. Elloumi, eds, Theory and Practice of Online Learning. Athabasca, Athabasca University

Anastasiades, P. (2009). Interactive Videoconferencing and Collaborative Distance Learning for K-12 Students and Teachers: Theory and Practice NY, Nova Science Publishers, Inc

Anastasiades, P. (2007). Interactive VideoConferencing (IVC) as a Crucial Factor in Distance Education: Towards a Constructivism IVC Pedagogy Model under a cross curricular thematic approach. In E. Bailey (Ed) Focus on Distance Education Developments., NY, Nova Science Publishers, Inc

Anastasiades, P. (2006). Interactive Videoconferencing in K- 9 Education: "ODUSSEAS 2000-2004" a case study in Elementary Schools in Greece and Cyprus. . Proceedings of the Diverse 2006, $6^{\text {th }}$ International Conference on Video and Videoconferencing in Education. 5-7 July, 2006, Scotland , Glasgow, Caledonian University

Anastasiades, P. (2003). Distance Learning in Elementary Schools in Cyprus: The evaluation Methodology and Results. Computers \& Education: volume. 40, no. 1, pp. 17-40, (24). Elsevier Science

Andrews, T. \& Klease, G. (1998). Challenges of multisite videoconferencing: The development of an alternative teaching/learning model. Australian Journal of Educational Technology, 14 (2), p. 88

Bean, J. (1997). The Middle School: The Nature Home of integrated curriculum. Educational Leadership, vol 49(2)

Bordwell, D. (1985). Narration in the Fiction Film. Madison: University of Wisconsin Press.0. Branigan, E. (1992). Narrative Comprehension and Film. London and New York, Routledge 
Brown, S. (2001). Views on Videoconferencing Higher Education and Research Opportunities in the UK (HERO), March $\quad$ Issues $\quad-\quad$ From http://www.hero.ac.uk/inside_he/archive/views_on_videoconferencin883.cfm?

Bruner, J. (1966). Toward a Theory of Instruction. Cambridge, Massachusetts: Harvard University Press.

Chandler, G., \& Hanrahan, P.. (2000). Teaching using interactive video: creating connections. Journal of Nursing Education 39 , pp. 73-80

Finn, K. E., Sellen, A. J., \& Wilbur, S. B. (Eds.). (1997). Video-mediated communication. Mahwah, NJ: Lawrence Erlbaum Associates. 15. Gibson, C., \& Cohen, S. (eds.) (2003). Virtual Teams That Work: Creating Conditions for Effective Virtual Teams. San Francisco. CA, Jossey- Bass/Wiley

Gürer, D., Kozma, R., \& Millán, E. (1999). Impact of shared applications and implications for the design of collaborative learning environments. In S. Lajoie (Ed.). Proceedings for the ninth world conference on artificial intelligence in education (AI-ED99, pp. 439-445). Amsterdam, IOS Press

Halas, J. \& Manvell, R. (1969). The technique of film animation. London and New York, Focal Press.

Laybourne, K., (1998). The Animation Book. New York, Three Rivers Press

Piaget, J. (1966). The Growth Of Logical Thinking from Childhood to Adolescence. London: Basic

Reed, J., \& Woodruff, M. (1995). An introduction to using videoconferencing technology for teaching, The Distance Educator Newsletter, Fall, Scott, T D and Pitcher, N (1997) SUMSMAN: Scottish, from: http://www.kn.pacbell.com/wired/vidconf/Using.html

Reisslein, J. Seeling, P. \& Reisslein, M. (2005). Video in distance education: ITES vs Web-Streaming: Evaluation of student attitudes. Internet and Higher Education, 8, pp. 25-44

Suthers, D. (2001). Collaborative representations: Supporting face to face and online knowledge-building discourse. Proceedings of the 34th Hawai 1 International Conference on the System Sciences (HICSS- 34), January 3-6, 2001, Maui, Hawai i: Institute of Electrical and Electronics Engineers.

Vygotsky, L.S. (1978). Mind and society: The development of higher mental processes. Cambridge, Harvard University Press

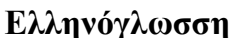

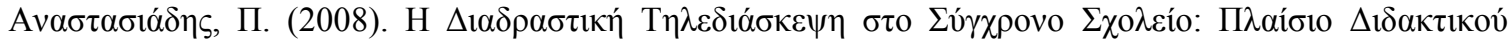

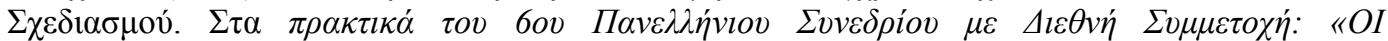

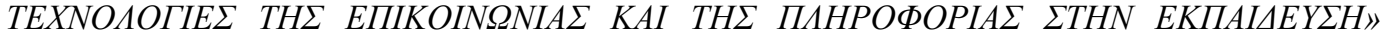
$\Lambda \varepsilon \mu \varepsilon \sigma o ́ \varsigma$ 25- $28 \Sigma \varepsilon \pi \tau \varepsilon \mu \beta$ píov 2008

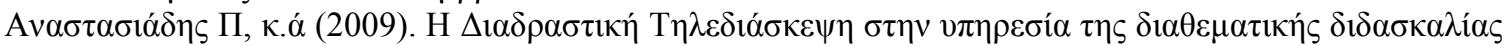

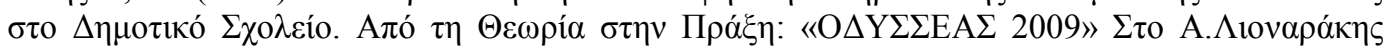

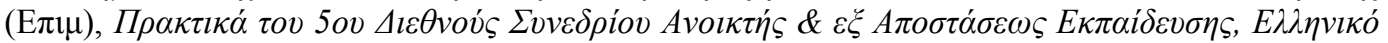

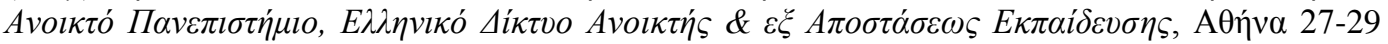

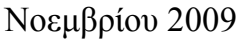

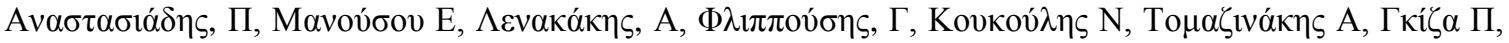

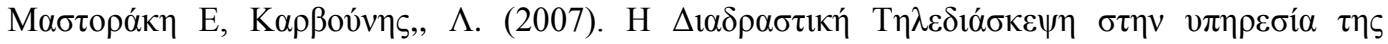

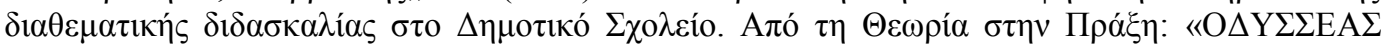

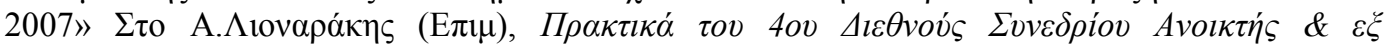

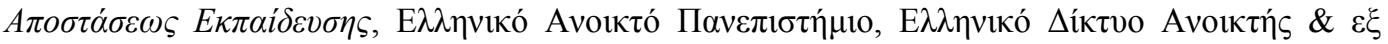

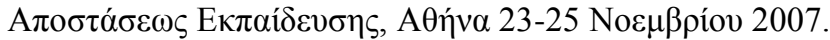

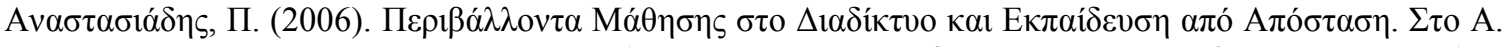

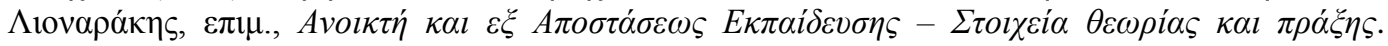
А $\theta \dot{v} v \alpha, \Pi \rho о \pi о \mu \pi$ ó

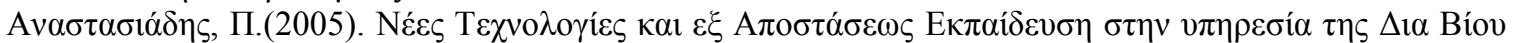

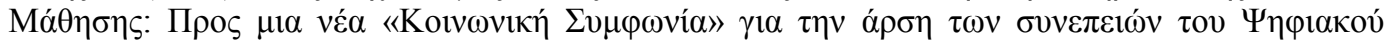

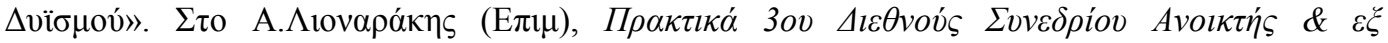

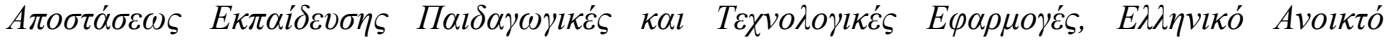

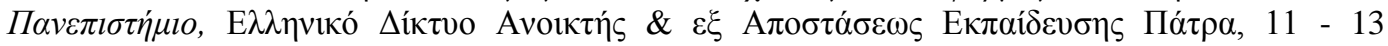

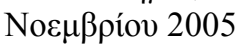

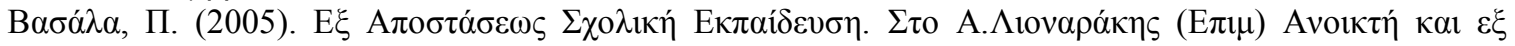

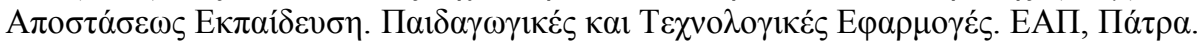

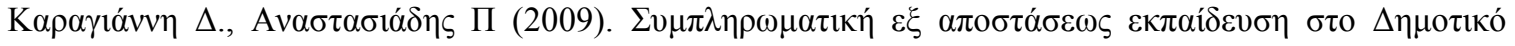

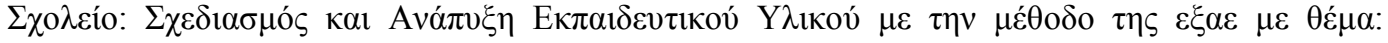




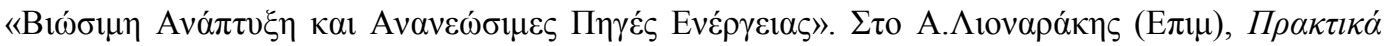

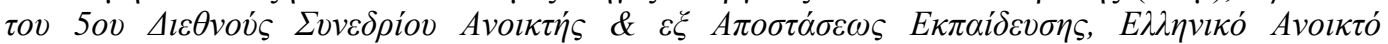

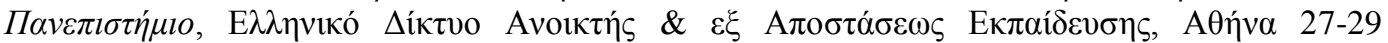

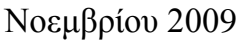

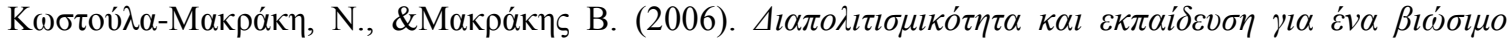
$\mu \dot{c} \lambda \lambda o v$. A $\theta \dot{v} v \alpha$

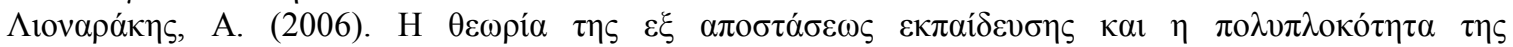

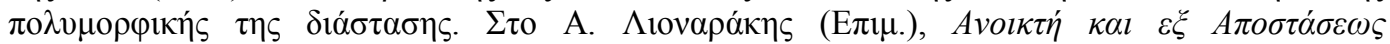

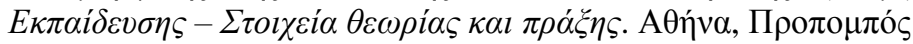

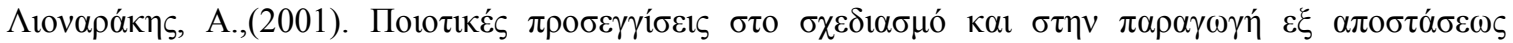

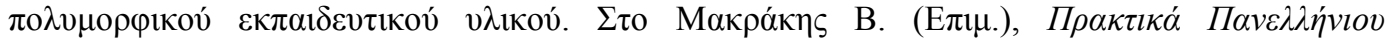

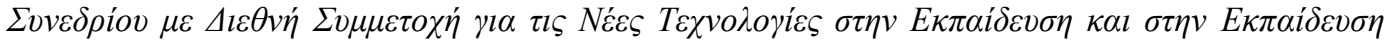

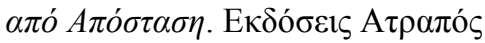

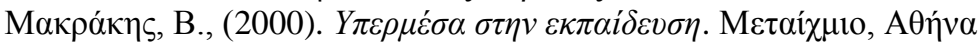

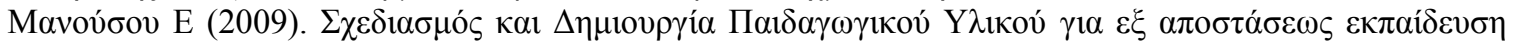

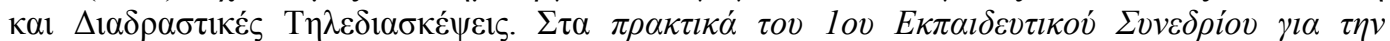

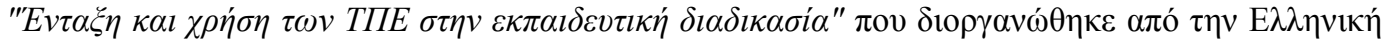

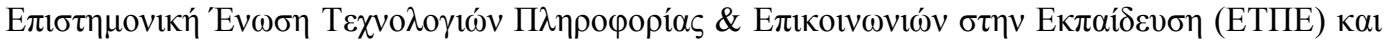

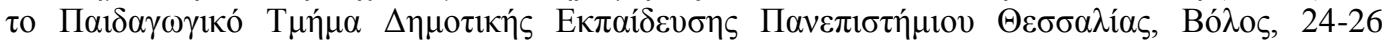
А

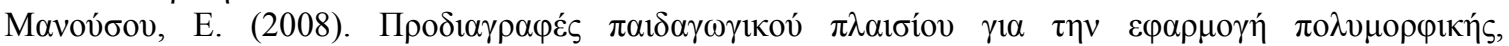

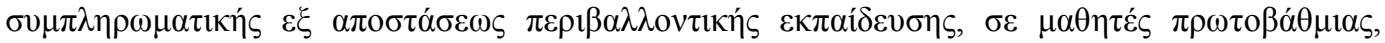

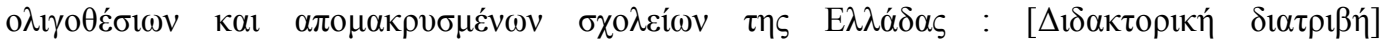
:http://193.108.161.35/cgi-bin-EL/egwcgi/167673/showfull.egw/ 1+0+1+full (15/2/2009)

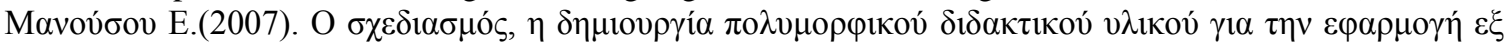

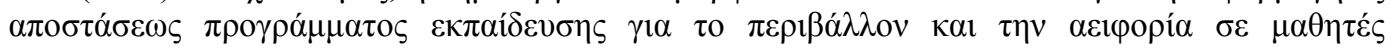

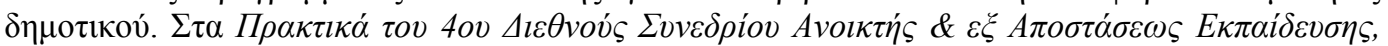

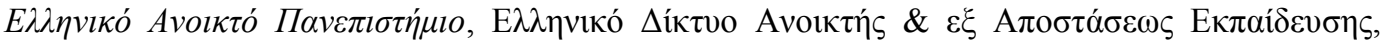

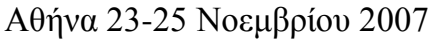

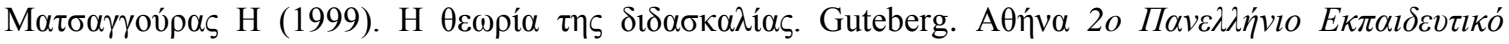

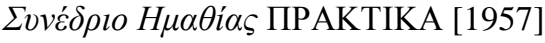

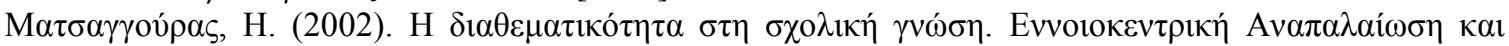

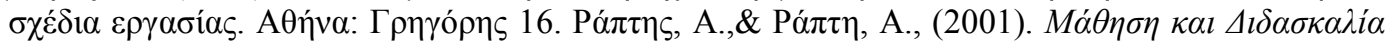

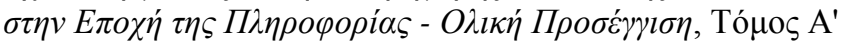

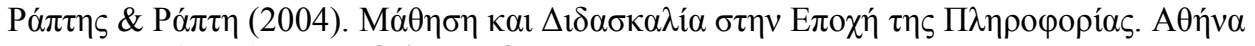

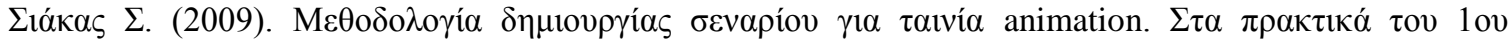

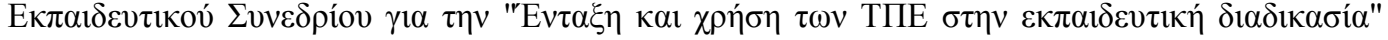

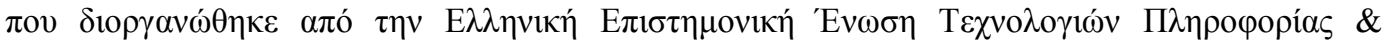

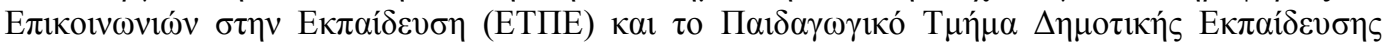

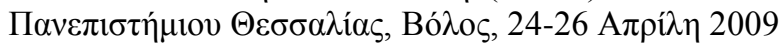

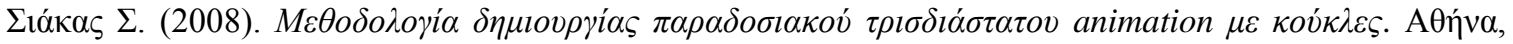

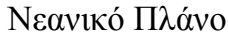

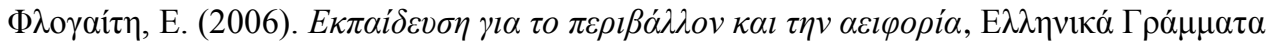

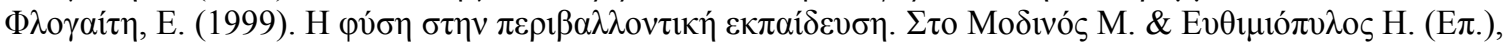

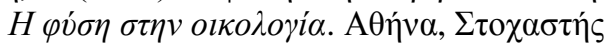

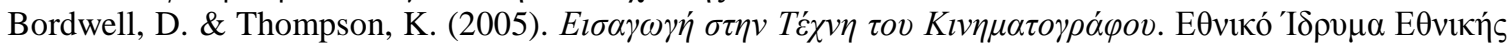

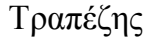

